QUARTERLY OF APPLIED MATHEMATICS

VOLUME LXVII, NUMBER 4

DECEMBER 2009, PAGES 627-660

S 0033-569X(09)01105-1

Article electronically published on May 5, 2009

\title{
SENSITIVITY EQUATIONS FOR A SIZE-STRUCTURED POPULATION MODEL
}

\author{
BY \\ H. T. BANKS (Center for Research in Scientific Computation, North Carolina State University, \\ Raleigh, NC 27695-8205), \\ STACEY L. ERNSTBERGER (Center for Research in Scientific Computation, North Carolina State \\ University, Raleigh, NC 27695-8205), \\ AND \\ SHUHUA HU (Center for Research in Scientific Computation, North Carolina State University, \\ Raleigh, NC 27695-8205)
}

\begin{abstract}
In this paper we consider the classical Sinko-Streifer size-structured population model and derive sensitivity partial differential equations for the sensitivities of solutions with respect to initial conditions, growth rate, mortality rate and fecundity rate. Sample numerical results to illustrate the use of these equations are also presented.
\end{abstract}

1. Introduction. Since the seminal work of Sinko and Streifer in [37] (see also [31] and the related efforts of McKendrick [33] and von Foerster [39]), size-structured population models and their generalizations have been widely investigated. Some of these efforts [3, 4, 29, 32] have focused on establishing well-posedness and stability analysis of models, formulating schemes for numerical solutions, and parameter estimation of individual rates. However, to the best of our knowledge, thus far there is no literature on sensitivity equations and the related analysis for size-structured population models. Sensitivity analysis of dynamical systems has drawn the attention of numerous researchers [1, 6, 9, 10, 11, 13, 14, 15, 16, 17, 20, 24, 25, 27, 28, 35, 38, 40] for many years because the resulting sensitivity functions can be used in many areas such as optimization and design [16, 26, 27, 34, 38, computation of standard errors [9, 10, 19, 21, 36], and information theory [12] related quantities (e.g., the Fisher information matrix) as well as control theory, parameter estimation and inverse problems [5, 8, 9, 10, 11, 40, 41. One of our motivations for investigating sensitivity for size-structured population models derives from our efforts reported in [7], where a shrimp biomass production system and a

Received October 11, 2007.

2000 Mathematics Subject Classification. Primary 93B35, 90C31, 92D25.

Key words and phrases. Size-structured population models, sensitivity equations, method of characteristics, renewal equations, finite difference schemes.

(C)2009 Brown University Reverts to public domain 28 years from publication 
related medical countermeasure production system currently under design and development is modeled in part by classical size-structured population models. The growth and mortality rates of the shrimp population are affected by several environmental factors such as temperature and salinity (e.g, [30, 42]). Hence, the sensitivity of such a model with respect to the growth and mortality rates is one important factor in optimizing the entire production system.

In this paper we derive and investigate the sensitivity equations for the linear sizestructured population model

$$
\begin{aligned}
& u_{t}(x, t)+(g(x) u(x, t))_{x}+m(x) u(x, t)=0, \\
& g(0) u(0, t)=\int_{0}^{\bar{x}} \beta(x) u(x, t) d x, \\
& u(x, 0)=u_{0}(x) .
\end{aligned}
$$

Here $(x, t) \in[0, \bar{x}] \times[0, T]$, the function $u(x, t)$ denotes the population density with size $x$ at time $t, g$ is the size-dependent individual growth rate, $m$ represents the size-dependent mortality rate, $\beta$ is the size-dependent reproduction or fecundity rate, and $u_{0}$ is the initial population density. The maximum size individuals may obtain in their lifetime is $\bar{x}$. The objective in this paper is to derive equations for the sensitivities of $u$ with respect to $g$, $m, \beta$, and $u_{0}$.

For traditional finite-dimensional parameter-dependent ordinary differential equation systems

$$
\dot{x}(t)=f(x(t), \theta), \quad x(0)=x_{0},
$$

one finds that the heuristic differentiation with respect to $\theta$ of the system (with an interchange of derivatives with respect to $t$ and $\theta$ and use of the chain rule) to obtain

$$
\dot{y}(t)=\frac{\partial f}{\partial x} y(t)+\frac{\partial f}{\partial \theta}, \quad y(0)=0,
$$

for $y(t)=\frac{\partial x}{\partial \theta}(t)$ provides the correct sensitivity equations. Moreover, this can be made completely rigorous, obtaining not only the form of the equations but also their wellposedness. While differentiation of (1) with respect to the functions $g, m, \beta$ or $u_{0}$ is somewhat more delicate, our goal is to establish that a formal, heuristic differentiation also results in the correct sensitivity equations and that this heuristic derivation can be made rigorous. As we shall see below, the underlying technical details are nontrivial. As we next outline, we considered several possible approaches before settling on the one employed here.

A semigroup approach is one of the popular and elegant methods used in the literature (e.g., [3, 4]) to establish the existence and uniqueness of solutions to size-structured population models. The idea behind this approach is to write the partial differential equation (11) in the abstract form

$$
\dot{u}(t)=\mathcal{A} u(t), \quad u(0)=u_{0},
$$

in a Banach space with the linear operator $\mathcal{A}$ defined by $\mathcal{A} \varphi=-(g \varphi)^{\prime}-m \varphi$. One can then show that $\mathcal{A}$ is an infinitesimal generator for a $C_{0}$-semigroup, and thereby establish existence and uniqueness of solutions to the model. One might then attempt to carry out 
a sensitivity analysis for (10) by using the abstract theoretical framework provided in [9], wherein the sensitivity analysis was developed for the general Banach space nonlinear ordinary differential equation

$$
\dot{z}(t)=f(t, z(t), \mu), \quad z\left(t_{0}\right)=z_{0} .
$$

Here the solution $z$ is in a complex Banach space $\mathcal{Z}$, the parameter $\mu$ is in a convex subset $\mathcal{M}$ of a topological vector space, and $f: \mathbb{R}_{+} \times \mathcal{Z} \times \mathcal{M} \rightarrow \mathcal{Z}$. However, our efforts in trying to apply this framework to (2) were not productive, as the domain of operator $\mathcal{A}$ is dependent on the parameters $g$ and $\beta$ themselves. For example, the domain of $\mathcal{A}$ in [4] is defined as

$$
\operatorname{dom} \mathcal{A}=\left\{\varphi \in \mathcal{H} \mid g \varphi \in \mathcal{H}^{1}(0, \bar{x}), \lim _{x \rightarrow \bar{x}}(g \varphi)(x)=0,(g \varphi)(0)=\int_{0}^{\bar{x}} \beta(x) \varphi(x) d x\right\},
$$

and $\mathcal{A}: \operatorname{dom} \mathcal{A} \subset \mathcal{H} \rightarrow \mathcal{H}$ for an appropriately defined Hilbert space $\mathcal{H}$.

The variational approach in 4] provides another method to establish existence and uniqueness of solutions to (11), where a Gelfand triple was constructed as $\mathcal{V} \hookrightarrow \mathcal{H} \hookrightarrow \mathcal{V}^{*}$ with $\mathcal{V}=\operatorname{dom} \mathcal{A}^{*}$. Here the adjoint operator $\mathcal{A}^{*}$ of $\mathcal{A}$ is given by

$$
\mathcal{A}^{*} \psi=g \psi^{\prime}-m \psi+\psi(0) \beta,
$$

with $\operatorname{dom} \mathcal{A}^{*}=\left\{\psi \in \mathcal{H} \mid \psi \in \mathcal{H}_{\mathrm{loc}}^{1}[0, \bar{x}), g \psi^{\prime} \in \mathcal{H}, \lim _{x \rightarrow \bar{x}}(g \varphi \psi)(x)=0\right.$ for $\left.\varphi \in \operatorname{dom} \mathcal{A}\right\}$, and the inner product on $\mathcal{V}$ is given by

$$
\langle\varphi, \psi\rangle_{\mathcal{V}}=\left\langle\left(\lambda_{0}-\mathcal{A}^{*}\right) \varphi,\left(\lambda_{0}-\mathcal{A}^{*}\right) \psi\right\rangle_{\mathcal{H}} .
$$

The variational form is then

$$
\begin{aligned}
& \frac{d}{d t}\langle u(t), \varphi\rangle_{\mathcal{V}^{*}, \mathcal{V}}=\left\langle u(t), \mathcal{A}^{*} \varphi\right\rangle_{\mathcal{H}} \text { for all } \varphi \in \mathcal{V}, t \geq 0 \\
& u(0)=u_{0} \in \mathcal{H}
\end{aligned}
$$

At first inspection it might appear more convenient to carry out a sensitivity analysis for (6) instead of (11) since all the model parameters are passed to the operator $\mathcal{A}^{*}$, which is linearly dependent on those parameters. However, the difficulty in using this approach is that $\mathcal{V}$ is chosen to be the domain of $\mathcal{A}^{*}$, and the resulting $\mathcal{V}$-norm is dependent on $g, m$, and $\beta$ (this can be seen from (4)-(5)). Hence, the sensitivity analysis of $u$ with respect to our model parameters is not readily carried out using either a semigroup or a variational approach because the domains of $\mathcal{A}$ and $\mathcal{A}^{*}$ are dependent on the model parameters. It might be possible to apply the idea of the method of mappings employed in sensitivity analysis for optimal shape design problems (e.g., [5, 22, 23, 26, 34]), which deals with computation of derivatives with respect to shape variation. However, it is not a straightforward application to our problem since the domain here is an operator domain (functional domain) instead of a geometric domain as used in optimal shape design problems.

The method of characteristics is another common technique used in the literature (e.g., see [3, 18, 29, 32]) to establish the existence and uniqueness of solutions to size-structured population models. Using this approach, one obtains an implicit representation of the solution which can be used to transform the partial differential equation into an integral equation. Then the contraction mapping theorem is applied in order to establish the 
desired results. In this paper we will employ this method to carry out a sensitivity analysis for (1). Although computationally tedious, this approach is conceptually straightforward and relatively easy to use.

The remainder of the paper is organized as follows. In Section 2, we give some preliminary theoretical results that are essential to our sensitivity derivations given in Section 3. Finite difference schemes to obtain numerical solutions of the model as well as the corresponding sensitivity equations are formulated in Section 4 , and some sample numerical results are also presented.

2. Preliminary results. In order to develop the sensitivity formulations of interest, a number of standing assumptions will be imposed on the model parameters and initial conditions in (1). We assume

(H1) $g \in \mathcal{W}^{1, \infty}(0, \bar{x}), g>0$ on $[0, \bar{x})$, and $g(\bar{x})=0$;

(H2) $m \in \mathcal{L}^{\infty}(0, \bar{x})$, and $m \geq 0$ on $[0, \bar{x}]$;

(H3) $\beta \in \mathcal{L}^{\infty}(0, \bar{x})$, and $\beta \geq 0$ on $[0, \bar{x}]$;

(H4) $u_{0} \in \mathcal{L}^{1}(0, \bar{x})$, and $u_{0} \geq 0$ on $[0, \bar{x}]$.

We will use $\|\cdot\|_{\infty}$ to denote the norm $\|\cdot\|_{\mathcal{L}^{\infty}(0, \bar{x})}$, and $\|\cdot\|_{1}$ to denote the norm $\|\cdot\|_{\mathcal{L}^{1}(0, \bar{x})}$ throughout the paper. Note that assumption (H1) implies that $g$ is in a convex subset of $\mathcal{W}^{1, \infty}(0, \bar{x})$; assumptions (H2) and (H3) guarantee that both $m$ and $\beta$ are in a convex subset of $\mathcal{L}^{\infty}(0, \bar{x})$, and assumption (H4) implies that $u_{0}$ is in a convex subset of $\mathcal{L}^{1}(0, \bar{x})$. Hence, we formulate the sensitivity equations for (1) by means of directional derivatives of $u$ with respect to the parameters $\beta, m, u_{0}$, and $g$. The directional derivative is defined by

Definition 2.1. Let $\Theta$ be a convex subset in some topological vector space, and let $f: \mathbb{R}_{+} \times \Theta \rightarrow R$. Given $\theta$ and $\vartheta$ in $\Theta$, we define the derivative $f_{\theta}(t ; \theta, \vartheta-\theta)$ of a function $f$ at $\theta$ in the direction $\vartheta-\theta$ to be

$$
f_{\theta}(t ; \theta, \vartheta-\theta)=\lim _{\epsilon \rightarrow 0+} \frac{f(t ; \theta+\epsilon(\vartheta-\theta))-f(t ; \theta)}{\epsilon},
$$

provided this limit exists.

The method of characteristics can be used to reduce equation (11) as well as each of the associated sensitivity equations to an equivalent renewal equation (a Volterra equation of convolution type), and the existence and uniqueness of solutions to these integral equations are established via the following result from [2] (Theorem 7.2 on page 220, and Theorem 7.4 on page 224).

RESult 2.1. If $\Psi(t)$ is bounded on $[0, T]$ and $\int_{0}^{T}|\phi(s)| d s<\infty$, then the equation

$$
\Phi(t)=\int_{0}^{t} \phi(s) \Phi(t-s) d s+\Psi(t)
$$

has a unique bounded solution in $[0, T]$. If we further assume that $\Psi(t)$ is continuous, then $\Phi(t)$ is also continuous. 
Suppose that the functions $\phi$ and $\Psi$ in equation (8) are both dependent on the parameter $\theta$ in a convex subset $\Theta$ of some topological space. Then equation (8) becomes

$$
\Phi(t ; \theta)=\int_{0}^{t} \phi(s ; \theta) \Phi(t-s ; \theta) d s+\Psi(t ; \theta) .
$$

We first give a sensitivity result for (9) because all our sensitivity results for (11) are heavily dependent upon it. We state this formally as a theorem because it is fundamental to the subsequent results in this paper.

Theorem 2.2. Suppose that for a given $\theta \in \Theta, \phi(t ; \theta)$ and $\Psi(t ; \theta)$ are both bounded on $[0, T]$. We assume further that for $\theta, \vartheta \in \Theta, \phi$ has a bounded directional derivative $\phi_{\theta}(t ; \theta, \vartheta-\theta)$ on $[0, T]$ with respect to $\theta \in \Theta$ in the direction $\vartheta-\theta$, and $\Psi$ has a bounded directional derivative $\Psi_{\theta}(t ; \theta, \vartheta-\theta)$ on $[0, T]$ with respect to $\theta \in \Theta$ in the direction $\vartheta-\theta$. Then the directional derivative $\Phi_{\theta}(t ; \theta, \vartheta-\theta)$ of $\Phi$ with respect to $\theta$ in the direction $\vartheta-\theta$ exists. Let $z(t)=\Phi_{\theta}(t ; \theta, \vartheta-\theta)$. Then it satisfies the following equation:

$$
z(t)=\int_{0}^{t} \phi(s ; \theta) z(t-s) d s+\int_{0}^{t} \phi_{\theta}(s ; \theta, \vartheta-\theta) \Phi(t-s ; \theta) d s+\Psi_{\theta}(t ; \theta, \vartheta-\theta) .
$$

We give arguments to establish these results. Note that the boundedness of $\phi(t ; \theta)$ and $\Psi(t ; \theta)$ on $[0, T]$ satisfy the assumptions of Result 2.1. Hence, the boundedness of $\Phi(t ; \theta)$ on $[0, T]$ is guaranteed. Since $\phi_{\theta}(t ; \theta, \vartheta-\theta)$ and $\Psi_{\theta}(t ; \theta, \vartheta-\theta)$ are both bounded on $[0, T]$, we know that there exists a positive constant $c$ such that

$$
\left|\int_{0}^{t} \phi_{\theta}(s ; \theta, \vartheta-\theta) \Phi(t-s ; \theta) d s+\Psi_{\theta}(t ; \theta, \vartheta-\theta)\right| \leq c \text { on }[0, T] .
$$

Thus, Result 2.1 guarantees that there exists a unique bounded solution $z(t)$ to (10). Note that

$$
\begin{aligned}
\Phi(t ; \theta+ & \epsilon(\vartheta-\theta))-\Phi(t ; \theta) \\
= & \int_{0}^{t} \phi(s ; \theta+\epsilon(\vartheta-\theta))[\Phi(t-s ; \theta+\epsilon(\vartheta-\theta))-\Phi(t-s ; \theta)] d s \\
& +\int_{0}^{t}[\phi(s ; \theta+\epsilon(\vartheta-\theta))-\phi(s ; \theta)] \Phi(t-s ; \theta) d s+\Psi(s ; \theta+\epsilon(\vartheta-\theta))-\Psi(s ; \theta) .
\end{aligned}
$$

Let $D(t ; \theta, \vartheta, \epsilon)=\frac{\Phi(t ; \theta+\epsilon(\vartheta-\theta))-\Phi(t ; \theta)}{\epsilon}-z(t)$. Then we find that

$$
\begin{aligned}
|D(t ; \theta, \vartheta, \epsilon)| \leq & \int_{0}^{t}|\phi(t-s ; \theta+\epsilon(\vartheta-\theta))||D(s ; \theta, \vartheta, \epsilon)| d s \\
& +\int_{0}^{t}|\phi(s ; \theta+\epsilon(\vartheta-\theta))-\phi(s ; \theta)||z(t-s)| d s \\
& +\int_{0}^{t}\left|\frac{\phi(s ; \theta+\epsilon(\vartheta-\theta))-\phi(s ; \theta)}{\epsilon}-\phi_{\theta}(s ; \theta, \vartheta-\theta)\right||\Phi(t-s ; \theta)| d s \\
& +\left|\frac{\Psi(t ; \theta+\epsilon(\vartheta-\theta))-\Psi(t ; \theta)}{\epsilon}-\Psi_{\theta}(t ; \theta, \vartheta-\theta)\right| .
\end{aligned}
$$


We observe that the boundedness of $\phi_{\theta}(t ; \theta, \vartheta-\theta)$ on $[0, T]$ implies that $\lim _{\epsilon \rightarrow 0+} \phi(t ; \theta+$ $\epsilon(\vartheta-\theta))=\phi(t ; \theta)$ uniformly in $t$. Hence, by the boundedness of $z$ we know that the second term on the right side of the above inequality converges to zero as $\epsilon \rightarrow 0$. The boundedness of $\Phi(t ; \theta)$ on $[0, T]$ and the existence of the directional derivative of $\phi$ with respect to $\theta$ in the direction $\vartheta-\theta$ imply that the third term on the right side of the above inequality converges to zero as $\epsilon \rightarrow 0$. The fourth term converges to zero as $\epsilon \rightarrow 0$ by the existence of the directional derivative of $\Psi$ with respect to $\theta$ in the direction $\vartheta-\theta$. Hence, by Gronwall's inequality we have

$$
\lim _{\epsilon \rightarrow 0+}|D(t ; \theta, \vartheta, \epsilon)|=0,
$$

and thus the theorem follows.

3. Sensitivity equations. In this section, we derive equations for the sensitivity of $u$ with respect to $\beta, m, u_{0}$ and $g$. First, we will use the method of characteristics to obtain the renewal equation for (11) and show that there exists a unique solution to this equation. We define several functions that will be used throughout this paper:

$$
G(x)=\int_{0}^{x} \frac{1}{g(\xi)} d \xi, \quad B(t)=\int_{0}^{\bar{x}} \beta(x) u(x, t) d x,
$$

where $u$ is the unique solution of (10) which is guaranteed to exist; see, e.g., [3, 29]. By assumption (H1), we find that for $\xi \in[0, \bar{x})$,

$$
0<g(\xi)=g(\xi)-g(\bar{x}) \leq\left\|g^{\prime}\right\|_{\infty}(\bar{x}-\xi) .
$$

Hence, we have

$$
G(x) \geq \frac{1}{\left\|g^{\prime}\right\|_{\infty}} \int_{0}^{x} \frac{1}{\bar{x}-\xi} d \xi
$$

which implies that $\lim _{x \rightarrow \bar{x}} G(x)=\infty$. Since $g>0$ on $[0, \bar{x}), G$ is a strictly increasing function so that $G^{-1}$ exists and is a strictly increasing map from $[0, \infty) \rightarrow[0, \bar{x})$. Therefore, the requirement of $g(\bar{x})=0$ in (H1) guarantees that the size of an individual always remains less than $\bar{x}$. In addition, to simplify the expressions, the following functions will be used throughout our derivations:

$$
\pi(x, t)=G^{-1}(G(x)-t), \varrho(t, \xi)=G^{-1}(t-\xi), \rho(x, t, \xi)=2 t-G(x)-\xi .
$$

Using the method of characteristics, we find that the solution of (1) is given implicitly by:

- If $x \geq G^{-1}(t)$, then

$$
u(x, t)=u_{0}\left(G^{-1}(G(x)-t)\right) \frac{g\left(G^{-1}(G(x)-t)\right)}{g(x)} \exp \left(-\int_{G^{-1}(G(x)-t)}^{x} \frac{m(\xi)}{g(\xi)} d \xi\right) .
$$

- If $x<G^{-1}(t)$, then

$$
u(x, t)=\frac{B(t-G(x))}{g(x)} \exp \left(-\int_{0}^{x} \frac{m(\xi)}{g(\xi)} d \xi\right) .
$$


Hence, we have

$$
\begin{aligned}
B(t)= & \int_{0}^{G^{-1}(t)} \beta(x) \frac{B(t-G(x))}{g(x)} \exp \left(-\int_{0}^{x} \frac{m(\xi)}{g(\xi)} d \xi\right) d x \\
& +\int_{G^{-1}(t)}^{\bar{x}} \beta(x) u_{0}(\pi(x, t)) \frac{g(\pi(x, t))}{g(x)} \exp \left(-\int_{\pi(x, t)}^{x} \frac{m(\xi)}{g(\xi)} d \xi\right) d x
\end{aligned}
$$

Let $\eta=G(x)$. Then $x=G^{-1}(\eta)$ and $d \eta=\frac{1}{g(x)} d x$. Hence, the first term on the right side of (13) can be rewritten as

$$
\begin{aligned}
\int_{0}^{G^{-1}(t)} & \beta(x) \frac{B(t-G(x))}{g(x)} \exp \left(-\int_{0}^{x} \frac{m(\xi)}{g(\xi)} d \xi\right) d x \\
= & \int_{0}^{t} \beta\left(G^{-1}(\eta)\right) \exp \left(-\int_{0}^{G^{-1}(\eta)} \frac{m(\xi)}{g(\xi)} d \xi\right) B(t-\eta) d \eta \\
= & \int_{0}^{t} \beta\left(G^{-1}(\eta)\right) \exp \left(-\int_{0}^{\eta} m\left(G^{-1}(\sigma)\right) d \sigma\right) B(t-\eta) d \eta .
\end{aligned}
$$

Let $\eta=G^{-1}(G(x)-t)$. Then $x=G^{-1}(G(\eta)+t)$ and $\frac{1}{g(\eta)} d \eta=\frac{1}{g(x)} d x$, which implies that $d x=\frac{g\left(G^{-1}(G(\eta)+t)\right)}{g(\eta)} d \eta$. Since $\lim _{\tau \rightarrow \infty} G^{-1}(\tau)=\bar{x}$, we have $\lim _{x \rightarrow \bar{x}} G^{-1}(G(x)-t)=\bar{x}$ for any $t \in[0, T]$. Hence, the second term on the right side of (13) can be rewritten as

$$
\begin{gathered}
\int_{G^{-1}(t)}^{\bar{x}} \beta(x) u_{0}\left(G^{-1}(G(x)-t)\right) \frac{g\left(G^{-1}(G(x)-t)\right)}{g(x)} \exp \left(-\int_{G^{-1}(G(x)-t)}^{x} \frac{m(\xi)}{g(\xi)} d \xi\right) d x \\
=\int_{0}^{\bar{x}} \beta\left(G^{-1}(G(\eta)+t)\right) u_{0}(\eta) \exp \left(-\int_{\eta}^{G^{-1}(G(\eta)+t)} \frac{m(\xi)}{g(\xi)} d \xi\right) d \eta
\end{gathered}
$$

Let $\sigma=G(\xi)-G(\eta)$. Then $\xi=G^{-1}(G(\eta)+\sigma)$ and $d \sigma=\frac{1}{g(\xi)} d \xi$. Hence, we have that

$$
\exp \left(-\int_{\eta}^{G^{-1}(G(\eta)+t)} \frac{m(\xi)}{g(\xi)} d \xi\right)=\exp \left(-\int_{0}^{t} m\left(G^{-1}(G(\eta)+\sigma)\right) d \sigma\right) .
$$

Thus, from the above equality and (15) we have

$$
\begin{gathered}
\int_{G^{-1}(t)}^{\bar{x}} \beta(x) u_{0}\left(G^{-1}(G(x)-t)\right) \frac{g\left(G^{-1}(G(x)-t)\right)}{g(x)} \exp \left(-\int_{G^{-1}(G(x)-t)}^{x} \frac{m(\xi)}{g(\xi)} d \xi\right) d x \\
=\int_{0}^{\bar{x}} u_{0}(\eta) \beta\left(G^{-1}(G(\eta)+t)\right) \exp \left(-\int_{0}^{t} m\left(G^{-1}(G(\eta)+\sigma)\right) d \sigma\right) d \eta
\end{gathered}
$$

Therefore, by (13), (14) and (16) we obtain the renewal equation

$$
B(t)=\int_{0}^{t} k(\eta) B(t-\eta) d \eta+F(t),
$$


where

$$
\begin{aligned}
& k(\eta)=\beta\left(G^{-1}(\eta)\right) \exp \left(-\int_{0}^{\eta} m\left(G^{-1}(\sigma)\right) d \sigma\right), \\
& F(t)=\int_{0}^{\bar{x}} u_{0}(\eta) \beta\left(G^{-1}(G(\eta)+t)\right) \exp \left(-\int_{0}^{t} m\left(G^{-1}(G(\eta)+\sigma)\right) d \sigma\right) d \eta .
\end{aligned}
$$

Note that $0 \leq F(t) \leq\|\beta\|_{\infty}\left\|u_{0}\right\|_{1}$, and $0 \leq k(t) \leq\|\beta\|_{\infty}$ on $[0, T]$. Hence, Result 2.1 guarantees that there exists a unique bounded nonnegative solution to (17). Thus, this solution of (17) must be the same as $B$ used in equations (11) and (12) in representing the unique nonnegative solution to (11).

We observe from equation (12) that the representation form for $u(x, t)$ in the region $\left\{(x, t) \mid 0 \leq x<G^{-1}(t), t>0\right\}$ is dependent on $B$. Hence, in order to find the sensitivity functions for $u$ with respect to $\beta, m, u_{0}$ and $g$, we first need to investigate sensitivity for $B$. To simplify the notation, we use $h$ to denote a given direction in the corresponding parameter space whenever we take a directional derivative with respect to either $\beta, m$, $u_{0}$ or $g$. In addition, we always suppress the parameter variables $\left(\beta, m, u_{0}\right.$ or $\left.g\right)$ in a function dependent on them unless it can cause confusion, such as when taking the derivative with respect to those parameters.

Since the sensitivity of $u$ with respect to $g$ is much more complicated than those with respect to $\beta, m$ or $u_{0}$, we will consider it subsequently in Section 3.4. At present our goal is to derive the sensitivity equations for $B$ with respect to $\beta, m$ and $u_{0}$. First we see that the directional derivative of $k$ with respect to $\beta$ in any given direction $h$ exists. Let $k_{\beta}(\eta ; \beta, h)$ denote the directional derivative of $k$ with respect to $\beta$ in the direction $h$. Then it is given by

$$
k_{\beta}(\eta ; \beta, h)=h\left(G^{-1}(\eta)\right) \exp \left(-\int_{0}^{\eta} m\left(G^{-1}(\sigma)\right) d \sigma\right),
$$

which implies that $\left|k_{\beta}(\eta ; \beta, h)\right| \leq\|h\|_{\infty}$ for $\eta \in[0, T]$. We also observe that the directional derivative of $F$ with respect to $\beta$ in any given direction $h$ exists. Let $F_{\beta}(t ; \beta, h)$ denote the derivative of $F$ with respect to $\beta$ in the direction $h$. Then it satisfies

$$
F_{\beta}(t ; \beta, h)=\int_{0}^{\bar{x}} h\left(G^{-1}(G(\eta)+t)\right) u_{0}(\eta) \exp \left(-\int_{0}^{t} m\left(G^{-1}(G(\eta)+\sigma)\right) d \sigma\right) d \eta,
$$

which implies that $\left|F_{\beta}(t ; \beta, h)\right| \leq\|h\|_{\infty}\left\|u_{0}\right\|_{1}$ for $t \in[0, T]$. Thus, by Theorem 2.2, the directional derivative $B_{\beta}(t ; \beta, h)$ of $B$ with respect to $\beta$ in the direction $h$ exists and is given by

$$
B_{\beta}(t ; \beta, h)=\int_{0}^{t} k(\eta) B_{\beta}(t-\eta ; \beta, h) d \eta+\int_{0}^{t} k_{\beta}(\eta ; \beta, h) B(t-\eta) d \eta+F_{\beta}(t ; \beta, h) .
$$

It also follows that there exists a unique bounded solution to equation (21).

Next we consider the sensitivity of $B$ with respect to $m$. By the chain rule we find that the directional derivative $k_{m}(\eta ; m, h)$ of $k$ with respect to $m$ in the direction $h$ satisfies

$$
\begin{aligned}
k_{m}(\eta ; m, h) & =-\beta\left(G^{-1}(\eta)\right) \exp \left(-\int_{0}^{\eta} m\left(G^{-1}(\sigma)\right) d \sigma\right)\left(\int_{0}^{\eta} h\left(G^{-1}(\sigma)\right) d \sigma\right) \\
& =-k(\eta)\left(\int_{0}^{\eta} h\left(G^{-1}(\sigma)\right) d \sigma\right),
\end{aligned}
$$


and the directional derivative $F_{m}(t ; m, h)$ of $F$ with respect to $m$ in the direction $h$ is given by

$$
\begin{aligned}
F_{m}(t ; m, h)= & -\int_{0}^{\bar{x}} u_{0}(\eta) \beta(\pi(\eta,-t)) \exp \left(-\int_{0}^{t} m(\pi(\eta,-\sigma)) d \sigma\right) \\
& \times\left(\int_{0}^{t} h(\pi(\eta,-\sigma)) d \sigma\right) d \eta .
\end{aligned}
$$

Hence, $\left|k_{m}(\eta ; m, h)\right| \leq T\|h\|_{\infty}\|\beta\|_{\infty}$ for $\eta \in[0, T]$ and $\left|F_{m}(t ; m, h)\right| \leq T\|h\|_{\infty}\|\beta\|_{\infty}\left\|u_{0}\right\|_{1}$ for $t \in[0, T]$. Thus, we know from Theorem 2.2 that the directional derivative $B_{m}(t ; m, h)$ of $B$ with respect to $m$ in the direction $h$ exists as the unique bounded solution to

$$
B_{m}(t ; m, h)=\int_{0}^{t} k(\eta) B_{m}(t-\eta ; m, h) d \eta+\int_{0}^{t} k_{m}(\eta ; m, h) B(t-\eta) d \eta+F_{m}(t ; m, h) .
$$

We observe that $k$ does not depend on $u_{0}$. Hence, the directional derivative $k_{u_{0}}\left(\eta ; u_{0}, h\right)$ of $k$ with respect to $u_{0}$ in the direction $h$ is given by

$$
k_{u_{0}}\left(\eta ; u_{0}, h\right)=0, \quad 0 \leq \eta \leq T .
$$

We also note that the directional derivative $F_{u_{0}}\left(t ; u_{0}, h\right)$ of $F$ with respect to $u_{0}$ in any given direction $h$ exists and is readily seen to be

$$
F_{u_{0}}\left(t ; u_{0}, h\right)=\int_{0}^{\bar{x}} h(\eta) \beta\left(G^{-1}(G(\eta)+t)\right) \exp \left(-\int_{0}^{t} m\left(G^{-1}(G(\eta)+\sigma)\right) d \sigma\right) d \eta,
$$

which implies that $\left|F_{u_{0}}\left(t ; u_{0}, h\right)\right| \leq\|\beta\|_{\infty}\|h\|_{1}$ for $t \in[0, T]$. Let $B_{u_{0}}\left(t ; u_{0}, h\right)$ denote the directional derivative of $B$ with respect to $u_{0}$ in the direction $h$. Then by Theorem 2.2 we know that it exists and is the unique bounded solution to

$$
B_{u_{0}}\left(t ; u_{0}, h\right)=\int_{0}^{t} k(\eta) B_{u_{0}}\left(t-\eta ; u_{0}, h\right) d \eta+F_{u_{0}}\left(t ; u_{0}, h\right) .
$$

Based on the above discussions on the sensitivities for $B$ with respect to $\beta, m$ and $u_{0}$, we can obtain the sensitivities for $u$ with respect to the same functions using the expressions for $u$ in (11) and (12). First we see that $u$ is independent of $\beta$ in the region $\left\{(x, t) \mid \bar{x}>x \geq G^{-1}(t), t \geq 0\right\}$. Hence, we see that the directional derivative $u_{\beta}(x, t ; \beta, h)$ of $u$ with respect to $\beta$ in the direction $h$ is zero in that region, that is,

$$
u_{\beta}(x, t ; \beta, h)=0, \text { for } x \geq G^{-1}(t) .
$$

Note that $B_{\beta}(t ; \beta, h)$ exists for $t \in[0, T]$. Hence, by (12) we find that

$$
u_{\beta}(x, t ; \beta, h)=\frac{B_{\beta}(t-G(x) ; \beta, h)}{g(x)} \exp \left(-\int_{0}^{x} \frac{m(\xi)}{g(\xi)} d \xi\right) \text {, for } x<G^{-1}(t) .
$$

By (11), (12), the existence of $B_{m}(t ; m, h)$ for $t \in[0, T]$ and the chain rule, we have that the directional derivative $u_{m}(x, t ; m, h)$ of $u$ with respect to $m$ in the direction $h$ satisfies

$$
u_{m}(x, t ; m, h)=-u(x, t) \int_{G^{-1}(G(x)-t)}^{x} \frac{h(\tau)}{g(\tau)} d \tau, \text { if } x \geq G^{-1}(t),
$$


and

$$
\begin{aligned}
u_{m}(x, t ; m, h)= & \frac{B_{m}(t-G(x) ; m, h)}{g(x)} \exp \left(-\int_{0}^{x} \frac{m(\tau)}{g(\tau)} d \tau\right) \\
& -u(x, t) \int_{0}^{x} \frac{h(\tau)}{g(\tau)} d \tau, \text { if } x<G^{-1}(t) .
\end{aligned}
$$

By (11), (12) and the existence of $B_{u_{0}}\left(t ; u_{0}, h\right)$ for $t \in[0, T]$, we find that if $x \geq G^{-1}(t)$, then we have

$$
u_{u_{0}}\left(x, t ; u_{0}, h\right)=h\left(G^{-1}(G(x)-t)\right) \frac{g\left(G^{-1}(G(x)-t)\right)}{g(x)} \exp \left(-\int_{G^{-1}(G(x)-t)}^{x} \frac{m(\xi)}{g(\xi)} d \xi\right),
$$

and if $x<G^{-1}(t)$, then we have

$$
u_{u_{0}}\left(x, t ; u_{0}, h\right)=\frac{B_{u_{0}}\left(t-G(x) ; u_{0}, h\right)}{g(x)} \exp \left(-\int_{0}^{x} \frac{m(\xi)}{g(\xi)} d \xi\right) .
$$

From the computational perspective, it is usually much easier to solve numerically a differential equation than an integral equation. Hence, in the next three subsections, we will show that $u_{\beta}(x, t ; \beta, h), u_{m}(x, t ; m, h)$ and $u_{u_{0}}\left(x, t ; u_{0}, h\right)$ are each respectively the solution to some first-order hyperbolic partial differential equation. This means that we can use these partial differential equations to obtain the numerical solutions for the sensitivity of $u$ with respect to these functions instead of using integral equations such as (24) coupled with (31). As we shall see, these partial differential equations are in each case precisely those that would be obtained if one simply heuristically differentiated equation (11) with respect to $\beta, m$ and $u_{0}$ respectively.

3.1. Sensitivity with respect to fecundity rate. In this subsection, we want to derive the sensitivity partial differential equation for $u_{\beta}$, the sensitivity of $u$ with respect to $\beta$. Let $v$ be the unique solution (guaranteed, for example, by results in Section 2 of [3] or a slight modification of the arguments for Proposition 2.2 of [29]) of the initial boundary value problem

$$
\begin{aligned}
& v_{t}(x, t)+(g(x) v(x, t))_{x}+m(x) v(x, t)=0, \\
& g(0) v(0, t)=\int_{0}^{\bar{x}}[\beta(x) v(x, t)+h(x) u(x, t)] d x, \\
& v(x, 0)=0 .
\end{aligned}
$$

Our aim here is to characterize the unique solution to (34) and to argue that $v=\frac{\partial u}{\partial \beta}[h]$, the directional derivative of $u$ with respect to $\beta$ in the direction $h$. Using the method of characteristics, we find that the implicit representation form for the solution to (34) is given as follows:

- If $x \geq G^{-1}(t)$, then

$$
v(x, t)=0 .
$$

- If $x<G^{-1}(t)$, then

$$
v(x, t)=\frac{V(t-G(x))}{g(x)} \exp \left(-\int_{0}^{x} \frac{m(\xi)}{g(\xi)} d \xi\right),
$$


Hence, we have

where $V(t)=\int_{0}^{\bar{x}}[\beta(x) v(x, t)+h(x) u(x, t)] d x$.

$$
\begin{aligned}
V(t)= & \int_{0}^{G^{-1}(t)} \beta(x) \frac{V(t-G(x))}{g(x)} \exp \left(-\int_{0}^{x} \frac{m(\xi)}{g(\xi)} d \xi\right) d x \\
& +\int_{0}^{G^{-1}(t)} h(x) \frac{B(t-G(x))}{g(x)} \exp \left(-\int_{0}^{x} \frac{m(\xi)}{g(\xi)} d \xi\right) d x \\
& +\int_{G^{-1}(t)}^{\bar{x}} h(x) u_{0}\left(G^{-1}(G(x)-t)\right) \frac{g\left(G^{-1}(G(x)-t)\right)}{g(x)} \\
& \times \exp \left(-\int_{G^{-1}(G(x)-t)}^{x} \frac{m(\xi)}{g(\xi)} d \xi\right) d x
\end{aligned}
$$

Using the same transformation as we derived for $B(t)$, we find that

$$
\begin{aligned}
V(t)= & \int_{0}^{t} \beta\left(G^{-1}(\eta)\right) \exp \left(-\int_{0}^{\eta} m\left(G^{-1}(\sigma)\right) d \sigma\right) V(t-\eta) d \eta \\
& +\int_{0}^{t} h\left(G^{-1}(\eta)\right) \exp \left(-\int_{0}^{\eta} m\left(G^{-1}(\sigma)\right) d \sigma\right) B(t-\eta) d \eta \\
& +\int_{0}^{\bar{x}} h\left(G^{-1}(G(\eta)+t)\right) u_{0}(\eta) \exp \left(-\int_{0}^{t} m\left(G^{-1}(G(\eta)+\sigma)\right) d \sigma\right) d \eta,
\end{aligned}
$$

which implies, by (19) and (20), that

$$
V(t)=\int_{0}^{t} k(\eta) V(t-\eta) d \eta+\int_{0}^{t} k_{\beta}(\eta ; \beta, h) B(t-\eta) d \eta+F_{\beta}(t ; \beta, h)
$$

Hence, equations (21), (38) and the uniqueness of the solution to (21) imply that

$$
V(t)=B_{\beta}(t ; \beta, h) \text { for any } t \in[0, T],
$$

and (35), (36) agree with the unique solution of (34).

Next we argue that $\frac{\partial u}{\partial \beta}[h]=v$, which means that $u_{\beta}(x, t ; \beta, h)$ satisfies the initial boundary value problem (34). By (28) and (35) we know that $u_{\beta}(x, t ; \beta, h)=v(x, t)$ in the region $\left\{(x, t) \mid \bar{x}>x \geq G^{-1}(t), t \geq 0\right\}$. From (36) and (39) we see that

$$
v(x, t)=\frac{B_{\beta}(t-G(x) ; \beta, h)}{g(x)} \exp \left(-\int_{0}^{x} \frac{m(\xi)}{g(\xi)} d \xi\right) .
$$

Hence, by the above equality and (29), we have that $u_{\beta}(x, t ; \beta, h)=v(x, t)$ in the region $\left\{(x, t) \mid 0 \leq x<G^{-1}(t), t>0\right\}$. Thus, $u_{\beta}(x, t ; \beta, h)$ satisfies the initial boundary value problem (34) and we can use this system to solve for $u_{\beta}(x, t ; \beta, h)$.

3.2. Sensitivity with respect to mortality rate. The sensitivity of $u$ with respect to $m$ is considered in this section. Let $w$ be the unique solution to the equation

$$
\begin{aligned}
& w_{t}(x, t)+(g(x) w(x, t))_{x}+m(x) w(x, t)+h(x) u(x, t)=0, \\
& g(0) w(0, t)=\int_{0}^{\bar{x}} \beta(x) w(x, t) d x, \\
& w(x, 0)=0 .
\end{aligned}
$$


We wish to show that $w=\frac{\partial u}{\partial m}[h]$, the directional derivative of $u$ with respect to $m$ in the direction $h$. Using the method of characteristics, we find that the solution to (40) is given by

- If $x \geq G^{-1}(t)$, then

$$
w(x, t)=-\int_{0}^{t} h(\pi(x, \xi)) u(\pi(x, \xi), t-\xi) \frac{g(\pi(x, \xi))}{g(x)} \exp \left(-\int_{\pi(x, \xi)}^{x} \frac{m(\tau)}{g(\tau)} d \tau\right) d \xi .
$$

- If $x<G^{-1}(t)$, then

$$
\begin{aligned}
w(x, t)= & \frac{W(t-G(x))}{g(x)} \exp \left(-\int_{0}^{x} \frac{m(\tau)}{g(\tau)} d \tau\right) \\
& -\int_{t-G(x)}^{t} h(\varrho(t, \xi)) u(\varrho(t, \xi), \rho(x, t, \xi)) \frac{g(\varrho(t, \xi))}{g(x)} \exp \left(-\int_{\varrho(t, \xi)}^{x} \frac{m(\tau)}{g(\tau)} d \tau\right) d \xi,
\end{aligned}
$$

$$
\text { where } W(t)=\int_{0}^{\bar{x}} \beta(x) w(x, t) d x .
$$

First we simplify the expression in (41). Note that $x \geq G^{-1}(t)$ implies that

$$
G(\pi(x, \xi))=G(x)-\xi \geq t-\xi .
$$

We further observe that $G^{-1}(G(\pi(x, \xi))-(t-\xi))=\pi(x, t)$. Hence, by (11) we find that

$$
u(\pi(x, \xi), t-\xi)=u_{0}(\pi(x, t)) \frac{g(\pi(x, t))}{g(\pi(x, \xi))} \exp \left(-\int_{\pi(x, t)}^{\pi(x, \xi)} \frac{m(\tau)}{g(\tau)} d \tau\right) .
$$

Thus, for the case $x \geq G^{-1}(t)$ we have that

$$
w(x, t)=-u_{0}(\pi(x, t)) \frac{g(\pi(x, t))}{g(x)} \exp \left(-\int_{\pi(x, t)}^{x} \frac{m(\tau)}{g(\tau)} d \tau\right) \int_{0}^{t} h(\pi(x, \xi)) d \xi .
$$

We simplify the expression in (42). Note that $x<G^{-1}(t)$ implies that

$$
G\left(G^{-1}(t-\xi)\right)=t-\xi<t-\xi+(t-G(x))=2 t-G(x)-\xi .
$$

Hence, using equation (12) and the fact that $2 t-G(x)-\xi-G\left(G^{-1}(t-\xi)\right)=t-G(x)$, we find that

$$
u\left(G^{-1}(t-\xi), 2 t-G(x)-\xi\right)=\frac{B(t-G(x))}{g\left(G^{-1}(t-\xi)\right)} \exp \left(-\int_{0}^{G^{-1}(t-\xi)} \frac{m(\tau)}{g(\tau)} d \tau\right) .
$$

Using the above equality and (42), for the case $x<G^{-1}(t)$ we have that

$$
\begin{aligned}
w(x, t)= & \frac{W(t-G(x))}{g(x)} \exp \left(-\int_{0}^{x} \frac{m(\tau)}{g(\tau)} d \tau\right) \\
& -\frac{B(t-G(x))}{g(x)} \exp \left(-\int_{0}^{x} \frac{m(\tau)}{g(\tau)} d \tau\right) \int_{t-G(x)}^{t} h\left(G^{-1}(t-\xi)\right) d \xi .
\end{aligned}
$$


By (44) and (46) we find that

$$
\begin{aligned}
W(t)= & \int_{0}^{G^{-1}(t)} \beta(x) \frac{W(t-G(x))}{g(x)} \exp \left(-\int_{0}^{x} \frac{m(\xi)}{g(\xi)} d \xi\right) d x \\
& -\int_{0}^{G^{-1}(t)} \beta(x) \frac{B(t-G(x))}{g(x)} \exp \left(-\int_{0}^{x} \frac{m(\xi)}{g(\xi)} d \xi\right)\left(\int_{t-G(x)}^{t} h\left(G^{-1}(t-\xi)\right) d \xi\right) d x \\
& -\int_{G^{-1}(t)}^{\bar{x}} \beta(x) u_{0}(\pi(x, t)) \frac{g(\pi(x, t))}{g(x)} \exp \left(-\int_{\pi(x, t)}^{x} \frac{m(\xi)}{g(\xi)} d \xi\right)\left(\int_{0}^{t} h(\pi(x, \xi)) d \xi\right) d x .
\end{aligned}
$$

Using the same transformation as we derived for $B(t)$, we obtain that

$$
\begin{aligned}
W(t)= & \int_{0}^{t} \beta\left(G^{-1}(\eta)\right) \exp \left(-\int_{0}^{\eta} m\left(G^{-1}(\sigma)\right) d \sigma\right) W(t-\eta) d \eta \\
& -\int_{0}^{t} \beta\left(G^{-1}(\eta)\right) \exp \left(-\int_{0}^{\eta} m\left(G^{-1}(\sigma)\right) d \sigma\right)\left(\int_{0}^{\eta} h\left(G^{-1}(\sigma)\right) d \sigma\right) B(t-\eta) d \eta \\
& -\int_{0}^{\bar{x}} u_{0}(\eta) \beta(\pi(\eta,-t)) \exp \left(-\int_{0}^{t} m(\pi(\eta,-\sigma)) d \sigma\right)\left(\int_{0}^{t} h(\pi(\eta,-\sigma)) d \sigma\right) d \eta .
\end{aligned}
$$

From the above equality, equation (22), and equation (23), we see that

$$
W(t)=\int_{0}^{t} k(\eta) W(t-\eta) d \eta+\int_{0}^{t} k_{m}(\eta ; m, h) B(t-\eta) d \eta+F_{m}(t ; m, h) .
$$

Hence, equations (24), (47) and the uniqueness of the solution to (24) imply that

$$
W(t)=B_{m}(t ; m, h) \quad \text { for any } t \in[0, T] .
$$

Next we argue that $\frac{\partial u}{m}[h]=w$, which means that $u_{m}(x, t ; m, h)$ satisfies (40). Let $\tau=G^{-1}(G(x)-\xi) ;$ then $d \xi=-\frac{1}{g(\tau)} d \tau$. Hence, we find that

$$
\int_{0}^{t} h\left(G^{-1}(G(x)-\xi)\right) d \xi=\int_{G^{-1}(G(x)-t)}^{x} \frac{h(\tau)}{g(\tau)} d \tau .
$$

From equations (11), (44) and (49) we observe that

$$
w(x, t)=-u(x, t) \int_{G^{-1}(G(x)-t)}^{x} \frac{h(\tau)}{g(\tau)} d \tau, \text { if } x \geq G^{-1}(t) .
$$

Therefore, by (30) and (50) we have $\frac{\partial u}{\partial m}[h]=w$ in the region $\left\{(x, t) \mid \bar{x}>x \geq G^{-1}(t)\right.$, $t \geq 0\}$. Let $\tau=G^{-1}(t-\xi)$; then $d \xi=-\frac{1}{g(\tau)} d \tau$. Hence, we find that

$$
\int_{t-G(x)}^{t} h\left(G^{-1}(t-\xi)\right) d \xi=\int_{0}^{x} \frac{h(\tau)}{g(\tau)} d \tau
$$


From equations (46), (48) and (51) we see that if $x<G^{-1}(t)$, then

$$
\begin{aligned}
w(x, t)= & \frac{B_{m}(t-G(x) ; m, h)}{g(x)} \exp \left(-\int_{0}^{x} \frac{m(\tau)}{g(\tau)} d \tau\right) \\
& -\frac{B(t-G(x))}{g(x)} \exp \left(-\int_{0}^{x} \frac{m(\xi)}{g(\xi)} d \xi\right) \int_{0}^{x} \frac{h(\tau)}{g(\tau)} d \tau .
\end{aligned}
$$

By (31) and (52) we have $\frac{\partial u}{\partial m}[h]=w$ when $x<G^{-1}(t)$. Therefore, $u_{m}(x, t ; m, h)$ satisfies the initial boundary value problem (40), and we can use this system to solve for $u_{m}(x, t ; m, h)$.

3.3. Sensitivity with respect to initial conditions. In this section, we derive the equation for the sensitivity function $u_{u_{0}}$. Let $r$ be the unique solution of the initial boundary value problem

$$
\begin{aligned}
& r_{t}(x, t)+(g(x) r(x, t))_{x}+m(x) r(x, t)=0, \\
& g(0) r(0, t)=\int_{0}^{\bar{x}} \beta(x) r(x, t) d x \\
& r(x, 0)=h(x) .
\end{aligned}
$$

We argue that $r=\frac{\partial u}{\partial u_{0}}[h]$, the directional derivative of $u$ with respect to $u_{0}$ in the direction $h$. By the method of characteristics, we find that the solution of (53) is given by

- If $x \geq G^{-1}(t)$, then

$r(x, t)=h\left(G^{-1}(G(x)-t)\right) \frac{g\left(G^{-1}(G(x)-t)\right)}{g(x)} \exp \left(-\int_{G^{-1}(G(x)-t)}^{x} \frac{m(\xi)}{g(\xi)} d \xi\right)$.

- If $x<G^{-1}(t)$, then

$$
r(x, t)=\frac{R(t-G(x))}{g(x)} \exp \left(-\int_{0}^{x} \frac{m(\xi)}{g(\xi)} d \xi\right),
$$

where $R(t)=\int_{0}^{\bar{x}} \beta(x) r(x, t) d x$.

Hence, we have

$$
\begin{aligned}
R(t)= & \int_{0}^{G^{-1}(t)} \beta(x) \frac{R(t-G(x))}{g(x)} \exp \left(-\int_{0}^{x} \frac{m(\xi)}{g(\xi)} d \xi\right) d x \\
& +\int_{G^{-1}(t)}^{\bar{x}} \beta(x) h\left(G^{-1}(G(x)-t)\right) \frac{g\left(G^{-1}(G(x)-t)\right)}{g(x)} \\
& \times \exp \left(-\int_{G^{-1}(G(x)-t)}^{x} \frac{m(\xi)}{g(\xi)} d \xi\right) d x .
\end{aligned}
$$

Using the same transformation as we derived for $B(t)$ we find that

$$
\begin{aligned}
R(t)= & \int_{0}^{t} \beta\left(G^{-1}(\eta)\right) \exp \left(-\int_{0}^{\eta} m\left(G^{-1}(\sigma)\right) d \sigma\right) R(t-\eta) d \eta \\
& +\int_{0}^{\bar{x}} h(\eta) \beta\left(G^{-1}(G(\eta)+t)\right) \exp \left(-\int_{0}^{t} m\left(G^{-1}(G(\eta)+\sigma)\right) d \sigma\right) d \eta .
\end{aligned}
$$


By (25), (26) and the above equality, we have

$$
R(t)=\int_{0}^{t} k(\eta) R(t-\eta) d \eta+F_{u_{0}}\left(t ; u_{0}, h\right)
$$

(recall that $k_{u_{0}}=0$ ). Hence, by (27), (157) and the uniqueness of solution to (27) we have

$$
R(t)=B_{u_{0}}\left(t ; u_{0}, h\right) \text { for any } t \in[0, T] .
$$

By (32), (154), (33), (55) and (158), we also know that $\frac{\partial u}{\partial u_{0}}[h]=r$. This means that $u_{u_{0}}\left(x, t ; u_{0}, h\right)$ satisfies (53) and we can use this system to solve for $u_{u_{0}}\left(x, t ; u_{0}, h\right)$.

3.4. Sensitivity with respect to $\mathrm{g}$. In this section, we investigate the sensitivity of $u$ with respect to $g$. As we shall see, our considerations follow the ideas in the previous sections for other sensitivities albeit with substantially more tedium in the details. Let $e$ satisfy uniquely the initial boundary value system

$$
\begin{aligned}
& e_{t}(x, t)+(g(x) e(x, t))_{x}+m(x) e(x, t)+(h(x) u(x, t))_{x}=0, \\
& g(0) e(0, t)+h(0) u(0, t)=\int_{0}^{\bar{x}} \beta(x) e(x, t) d x, \\
& e(x, 0)=0 .
\end{aligned}
$$

Our goal is to argue that $e=\frac{\partial u}{\partial g}[h]$, the directional derivative of $u$ with respect to $g$ in the direction $h$. In order to carry out the necessary computations, we strengthen the conditions on $g, m, \beta$ and $u_{0}$ by assuming

(A1) $g \in \mathcal{C}^{1}(0, \bar{x}), g>0$ on $[0, \bar{x})$, and $g(\bar{x})=0$;

(A2) $m \in \mathcal{C}^{1}(0, \bar{x}), m \geq 0$ on $[0, \bar{x}]$

(A3) $\beta \in \mathcal{C}^{1}(0, \bar{x}), \beta \geq 0$ on $[0, \bar{x}]$;

(A4) $u_{0} \in \mathcal{C}^{1}(0, \bar{x}), u_{0} \geq 0$ on $[0, \bar{x}]$;

(A5) $u_{0}$ satisfies the compatibility condition

$$
g(0) u_{0}(0)=\int_{0}^{\bar{x}} \beta(x) u_{0}(x) d x .
$$

The notation $\|\cdot\|_{\mathcal{C}}$ will be used throughout this section to denote the supremum norm $\|\cdot\|_{\mathcal{C}(0, \bar{x})}$. We first establish that the directional derivative $u_{g}(x, t ; g, h)$ of $u$ at $g$ in any given direction $h$ exists, and then we will derive the solution $e(x, t)$ to (59) via the method of characteristics and argue that $\frac{\partial u}{\partial g}[h]=e$.

By assumption (A1), we know that $G(x)$ is twice continuously differentiable on $[0, \bar{x})$, which implies that $G^{-1}$ is twice continuously differentiable on $[0, \infty)$. Hence, $\pi(x, t)$ is twice continuously differentiable on $\left\{(x, t) \mid \bar{x}>x \geq G^{-1}(t), t \geq 0\right\}$. We observe that $G(\pi(x, t))=G(x)-t$. Hence, we have $G^{\prime}(\pi(x, t)) \pi_{x}(x, t)=G^{\prime}(x)$. Furthermore, we see that

Hence, we obtain that

$$
G^{\prime}(x)=\frac{1}{g(x)}
$$

$$
\pi_{x}(x, t)=\frac{g(\pi(x, t))}{g(x)}
$$


The directional derivative $G_{g}(x ; g, h)$ of $G$ at $g$ in any given direction $h$ exists and is given by

$$
G_{g}(x ; g, h)=-\int_{0}^{x} \frac{h(\tau)}{g^{2}(\tau)} d \tau
$$

Moreover, we find that

$$
G^{\prime}(\pi(x, t)) \pi_{g}(x, t ; g, h)+G_{g}(\pi(x, t) ; g, h)=G_{g}(x ; g, h),
$$

where $\pi_{g}(x, t ; g, h)$ denotes the directional derivative $\pi$ at $g$ in the given direction $h$. Thus, using equations (61) and (63) and the above equality, we obtain

$$
\pi_{g}(x, t ; g, h)=-g(\pi(x, t)) \int_{\pi(x, t)}^{x} \frac{h(\tau)}{g^{2}(\tau)} d \tau
$$

Let

$$
\varpi(x, t)=\exp \left(-\int_{\pi(x, t)}^{x} \frac{m(\xi)}{g(\xi)} d \xi\right) .
$$

Then by the chain rule and (64) we know that the derivative $\varpi_{g}(x, t ; g, h)$ of $\varpi$ at $g$ in any given direction $h$ exists and is given by

$$
\begin{aligned}
& \varpi_{g}(x, t ; g, h) \\
& \quad=\exp \left(-\int_{\pi(x, t)}^{x} \frac{m(\xi)}{g(\xi)} d \xi\right)\left[\frac{m(\pi(x, t))}{g(\pi(x, t))} \pi_{g}(x, t ; g, h)+\int_{\pi(x, t)}^{x} \frac{m(\xi) h(\xi)}{g^{2}(\xi)} d \xi\right] \\
& \quad=\exp \left(-\int_{\pi(x, t)}^{x} \frac{m(\xi)}{g(\xi)} d \xi\right)\left[\begin{array}{l}
\left.x(\pi(x, t)) \int_{\pi(x, t)}^{x} \frac{h(\tau)}{g^{2}(\tau)} d \tau+\int_{\pi(x, t)}^{x} \frac{m(\xi) h(\xi)}{g^{2}(\xi)} d \xi\right] .
\end{array}\right.
\end{aligned}
$$

Let $\chi(x, t)=\frac{g\left(G^{-1}(G(x)-t)\right)}{g(x)}$. Then by assumption (A1), the chain rule and (64) we know that the directional derivative $\chi_{g}(x, t ; g, h)$ of $\chi$ at $g$ in any given direction $h$ exists and is given by

$$
\begin{aligned}
\chi_{g}(x, t ; g, h) & =\frac{g^{\prime}(\pi(x, t))}{g(x)} \pi_{g}(x, t ; g, h)+\frac{h(\pi(x, t))}{g(x)}-\frac{g(\pi(x, t)) h(x)}{g^{2}(x)} \\
& =-\frac{g^{\prime}(\pi(x, t))}{g(x)} g(\pi(x, t)) \int_{\pi(x, t)}^{x} \frac{h(\tau)}{g^{2}(\tau)} d \tau+\frac{h(\pi(x, t))}{g(x)}-\frac{g(\pi(x, t)) h(x)}{g^{2}(x)} .
\end{aligned}
$$


Thus, assumption (A4) and equations (64) imply that $u_{g}(x, t ; g, h)$ exists in the region $\left\{(x, t) \mid \bar{x}>x \geq G^{-1}(t), t \geq 0\right\}$ and is given by

$$
\begin{aligned}
u_{g}(x, t ; g, h)= & -u_{0}^{\prime}(\pi(x, t)) \frac{g^{2}(\pi(x, t))}{g(x)} \exp \left(-\int_{\pi(x, t)}^{x} \frac{m(\xi)}{g(\xi)} d \xi\right) \int_{\pi(x, t)}^{x} \frac{h(\tau)}{g^{2}(\tau)} d \tau \\
= & -u_{0}(\pi(x, t)) \frac{g^{\prime}(\pi(x, t))}{g(x)} g(\pi(x, t)) \exp \left(-\int_{\pi(x, t)}^{x} \frac{m(\xi)}{g(\xi)} d \xi\right) \int_{\pi(x, t)}^{x} \frac{h(\tau)}{g^{2}(\tau)} d \tau \\
& +u_{0}(\pi(x, t)) \exp \left(-\int_{\pi(x, t)}^{x} \frac{m(\xi)}{g(\xi)} d \xi\right)\left[\frac{h(\pi(x, t))}{g(x)}-\frac{g(\pi(x, t)) h(x)}{g^{2}(x)}\right] \\
& -u_{0}(\pi(x, t)) \frac{g(\pi(x, t))}{g(x)} \exp \left(-\int_{\pi(x, t)}^{x} \frac{m(\xi)}{g(\xi)} d \xi\right)\left[m(\pi(x, t)) \int_{\pi(x, t)}^{x} \frac{h(\tau)}{g^{2}(\tau)} d \tau\right] \\
& +u_{0}(\pi(x, t)) \frac{g(\pi(x, t))}{g(x)} \exp \left(-\int_{\pi(x, t)}^{x} \frac{m(\xi)}{g(\xi)} d \xi\right)\left[\int_{\pi(x, t)}^{x} \frac{h(\xi) m(\xi)}{g^{2}(\xi)} d \xi\right] .
\end{aligned}
$$

Before we consider the sensitivity of $u$ with respect to $g$ in the region $\{(x, t) \mid 0 \leq x<$ $\left.G^{-1}(t), t>0\right\}$, we need to carry out a sensitivity analysis for the renewal equation (17) with respect to $g$. This is because from (12), $u$ is dependent on $B$ in this region. First we need to show that $F$ of (18) has a bounded directional derivative with respect to $g$. By equation (64), we have

$$
\pi_{g}(\eta,-t ; g, h)=g(\pi(\eta,-t)) \int_{\eta}^{\pi(\eta,-t)} \frac{h(\tau)}{g^{2}(\tau)} d \tau .
$$

Observe that $\pi(\eta,-t)=G^{-1}(G(\eta)+t)$. Hence, using assumptions (A2) and (A3), the chain rule and equation (68), we know that the directional derivative $F_{g}(t ; g, h)$ of $F$ at $g$ in any given direction $h$ exists and is given as follows:

$$
\begin{gathered}
F_{g}(t ; g, h)=\int_{0}^{\bar{x}} u_{0}(\eta) \beta^{\prime}(\pi(\eta,-t)) g(\pi(\eta,-t))\left(\int_{\eta}^{\pi(\eta,-t)} \frac{h(\tau)}{g^{2}(\tau)} d \tau\right) \\
\times \exp \left(-\int_{0}^{t} m(\pi(\eta,-\sigma)) d \sigma\right) d \eta \\
-\int_{0}^{\bar{x}} u_{0}(\eta) \beta(\pi(\eta,-t)) \exp \left(-\int_{0}^{t} m(\pi(\eta,-\sigma)) d \sigma\right) \\
\times\left[\int_{0}^{t} m^{\prime}(\pi(\eta,-\sigma)) \pi_{g}(\eta,-\sigma ; g, h) d \sigma\right] d \eta .
\end{gathered}
$$

Note that $\lim _{\eta \rightarrow \bar{x}} \pi(\eta,-t)=\bar{x}$ for any $t \in[0, T]$. Hence, if we define $\pi(\bar{x},-t)=\bar{x}$, then $\pi(\eta,-t)$ is continuous on $[0, \bar{x}] \times[0, T]$. Thus, from equation (68) and the continuity of $g$ and $h$, we know that $\pi_{g}(\eta,-t ; g, h)$ is continuous on $[0, \bar{x}] \times[0, T]$. Therefore, there exists a positive constant $\nu$ such that $\left|\pi_{g}(\eta,-t ; g, h)\right| \leq \nu$ for $(\eta, t) \in[0, \bar{x}] \times[0, T]$. Hence, by equation (69) we have that

$$
\left|F_{g}(t ; g, h)\right| \leq \nu \bar{x}\left\|u_{0}\right\|_{C}\left(\left\|\beta^{\prime}\right\|_{C}+T\|\beta\|_{C}\left\|m^{\prime}\right\|_{C}\right) \quad \text { for } t \in[0, T] .
$$


Next we show that $k$ has a bounded directional derivative with respect to $g$. Let $\kappa(\eta)=$ $G^{-1}(\eta)$. Then $G(\kappa(\eta))=\eta$. Hence, we find that

$$
G^{\prime}(\kappa(\eta)) \kappa_{g}(\eta ; g, h)+G_{g}(\kappa(\eta) ; g, h)=0,
$$

where $\kappa_{g}(\eta ; g, h)$ denotes the directional derivative of $\kappa$ at $g$ in the direction $h$. Using the above equality and equation (63), we obtain that

$$
\kappa_{g}(\eta ; g, h)=g(\kappa(\eta)) \int_{0}^{\kappa(\eta)} \frac{h(\tau)}{g^{2}(\tau)} d \tau
$$

By assumption (A3), the chain rule and equation (70), we have that the directional derivative $k_{g}(\eta ; g, h)$ of $k$ at $g$ in any given direction $h$ exists and is given by

$$
\begin{aligned}
k_{g}(\eta ; g, h)= & \beta^{\prime}\left(G^{-1}(\eta)\right) g\left(G^{-1}(\eta)\right)\left(\int_{0}^{G^{-1}(\eta)} \frac{h(\tau)}{g^{2}(\tau)} d \tau\right) \exp \left(-\int_{0}^{\eta} m\left(G^{-1}(\sigma)\right) d \sigma\right) \\
& -\beta\left(G^{-1}(\eta)\right) \exp \left(-\int_{0}^{\eta} m\left(G^{-1}(\sigma)\right) d \sigma\right)\left[\int_{0}^{\eta} m^{\prime}\left(G^{-1}(\sigma)\right) \kappa_{g}(\sigma ; g, h) d \sigma\right] .
\end{aligned}
$$

Observing that $G^{-1}(T)<\bar{x}$, from equation (70) we have

$$
\left|\kappa_{g}(\eta ; g, h)\right| \leq \bar{x}\|g\|_{C}\left\|h / g^{2}\right\|_{C\left(0, G^{-1}(T)\right)} \quad \text { for } \eta \in[0, T] .
$$

By this bound and (71) we obtain

$$
\left|k_{g}(\eta ; g, h)\right| \leq \bar{x}\left(\left\|\beta^{\prime}\right\|_{C}+T\|\beta\|_{C}\left\|m^{\prime}\right\|_{C}\right)\|g\|_{C}\left\|h / g^{2}\right\|_{C\left(0, G^{-1}(T)\right)} \quad \text { for } \eta \in[0, T] .
$$

Therefore, Theorem 2.2 implies that the directional derivative $B_{g}(t ; g, h)$ of $B$ at $g$ in any given direction $h$ exists and is the unique bounded solution of

$$
B_{g}(t ; g, h)=\int_{0}^{t} k(\eta) B_{g}(t-\eta ; g, h) d \eta+\int_{0}^{t} k_{g}(\eta ; g, h) B(t-\eta) d \eta+F_{g}(t ; g, h) .
$$

Note that assumptions (A1)-(A4) imply that both $k$ and $F$ are continuously differentiable on $[0, T]$. Hence, $B$ is continuously differentiable on $[0, T]$. Using the chain rule and equation (63), we have that $u_{g}(x, t ; g, h)$ exists in the region $\left\{(x, t) \mid 0 \leq x<G^{-1}(t)\right.$, $t>0\}$ and is given by

$$
\begin{aligned}
u_{g}(x, t ; g, h)= & \frac{B_{g}(t-G(x) ; g, h)}{g(x)} \exp \left(-\int_{0}^{x} \frac{m(\xi)}{g(\xi)} d \xi\right) \\
& +\frac{B^{\prime}(t-G(x))}{g(x)} \exp \left(-\int_{0}^{x} \frac{m(\xi)}{g(\xi)} d \xi\right)\left(\int_{0}^{x} \frac{h(\xi)}{g^{2}(\xi)} d \xi\right) \\
& -\frac{B(t-G(x))}{g^{2}(x)} h(x) \exp \left(-\int_{0}^{x} \frac{m(\xi)}{g(\xi)} d \xi\right) \\
& +\frac{B(t-G(x))}{g(x)} \exp \left(-\int_{0}^{x} \frac{m(\xi)}{g(\xi)} d \xi\right)\left(\int_{0}^{x} \frac{h(\xi) m(\xi)}{g^{2}(\xi)} d \xi\right) .
\end{aligned}
$$

Finally we show that $\frac{\partial u}{\partial g}[h]=e$, which yields that $u_{g}(x, t ; g, h)$ satisfies the initial boundary value problem (59). Using the method of characteristics, we find that the solution to (59) is given by 
- If $x \geq G^{-1}(t)$, then

$$
\begin{aligned}
e(x, t)= & -\int_{0}^{t} h^{\prime}(\pi(x, \xi)) u(\pi(x, \xi), t-\xi) \frac{g(\pi(x, \xi))}{g(x)} \exp \left(-\int_{\pi(x, \xi)}^{x} \frac{m(\tau)}{g(\tau)} d \tau\right) d \xi \\
& -\int_{0}^{t} h(\pi(x, \xi)) u_{1}(\pi(x, \xi), t-\xi) \frac{g(\pi(x, \xi))}{g(x)} \exp \left(-\int_{\pi(x, \xi)}^{x} \frac{m(\tau)}{g(\tau)} d \tau\right) d \xi
\end{aligned}
$$

where $u_{1}=\frac{\partial u}{\partial x}$ denotes the first partial derivative of $u$ with respect to $x$.

- If $x<G^{-1}(t)$, then

$$
\begin{aligned}
e(x, t)= & {\left[\frac{E(t-G(x))}{g(x)}-\frac{h(0)}{g(0)} \frac{B(t-G(x))}{g(x)}\right] \exp \left(-\int_{0}^{x} \frac{m(\tau)}{g(\tau)} d \tau\right) } \\
& -\int_{t-G(x)}^{t} h^{\prime}(\varrho(t, \xi)) u(\varrho(t, \xi), \rho(x, t, \xi)) \frac{g(\varrho(t, \xi))}{g(x)} \exp \left(-\int_{\varrho(t, \xi)}^{x} \frac{m(\tau)}{g(\tau)} d \tau\right) d \xi \\
& -\int_{t-G(x)}^{t} h(\varrho(t, \xi)) u_{1}(\varrho(t, \xi), \rho(x, t, \xi)) \frac{g(\varrho(t, \xi))}{g(x)} \exp \left(-\int_{\varrho(t, \xi)}^{x} \frac{m(\tau)}{g(\tau)} d \tau\right) d \xi,
\end{aligned}
$$

where $E(t)=\int_{0}^{\bar{x}} \beta(x) e(x, t) d x$.

We simplify the expression in (74). Note that $x \geq G^{-1}(t)$ implies that

$$
G(\pi(x, \xi))=G(x)-\xi \geq t-\xi .
$$

Let $\tau=G^{-1}(G(x)-\xi)$; then $d \xi=-\frac{1}{g(\tau)} d \tau$. Hence, we have

$$
\int_{0}^{t} h^{\prime}(\pi(x, \xi)) d \xi=\int_{\pi(x, t)}^{x} \frac{h^{\prime}(\tau)}{g(\tau)} d \tau
$$

Thus, using equation (43), we can rewrite the first term on the right side of (74) as

$$
\begin{aligned}
& \int_{0}^{t} h^{\prime}(\pi(x, \xi)) u(\pi(x, \xi), t-\xi) \frac{g(\pi(x, \xi))}{g(x)} \exp \left(-\int_{\pi(x, \xi)}^{x} \frac{m(\tau)}{g(\tau)} d \tau\right) d \xi \\
& =u_{0}(\pi(x, t)) \frac{g(\pi(x, t))}{g(x)} \exp \left(-\int_{\pi(x, t)}^{x} \frac{m(\tau)}{g(\tau)} d \tau\right)\left(\int_{\pi(x, t)}^{x} \frac{h^{\prime}(\tau)}{g(\tau)} d \tau\right)
\end{aligned}
$$


Note that assumptions (A1), (A2) and (A4) imply that $u$ is continuously differentiable in the region $\left\{(x, t) \mid \bar{x}>x \geq G^{-1}(t), t \geq 0\right\}$. Thus, by equation (62) we find that

$$
\begin{aligned}
u_{x}(x, t)= & u_{0}^{\prime}(\pi(x, t)) \frac{g^{2}(\pi(x, t))}{g^{2}(x)} \exp \left(-\int_{\pi(x, t)}^{x} \frac{m(\xi)}{g(\xi)} d \xi\right) \\
& +u_{0}(\pi(x, t)) \frac{g^{\prime}(\pi(x, t))}{g(x)} \frac{g(\pi(x, t))}{g(x)} \exp \left(-\int_{\pi(x, t)}^{x} \frac{m(\xi)}{g(\xi)} d \xi\right) \\
& -u_{0}(\pi(x, t)) \frac{g(\pi(x, t)) g^{\prime}(x)}{g^{2}(x)} \exp \left(-\int_{\pi(x, t)}^{x} \frac{m(\xi)}{g(\xi)} d \xi\right) \\
& +u_{0}(\pi(x, t)) \frac{g(\pi(x, t))}{g(x)} \exp \left(-\int_{\pi(x, t)}^{x} \frac{m(\xi)}{g(\xi)} d \xi\right)\left(\frac{m(\pi(x, t))}{g(x)}\right) \\
& -u_{0}(\pi(x, t)) \frac{g(\pi(x, t))}{g(x)} \exp \left(-\int_{\pi(x, t)}^{x} \frac{m(\xi)}{g(\xi)} d \xi\right)\left(\frac{m(x)}{g(x)}\right) .
\end{aligned}
$$

Observe that $G^{-1}(G(\pi(x, \xi))-(t-\xi))=G^{-1}(G(x)-t)=\pi(x, t)$. Hence, we have that

$$
\begin{aligned}
u_{1}(\pi(x, \xi), t-\xi)= & u_{0}^{\prime}(\pi(x, t)) \frac{g^{2}(\pi(x, t))}{g^{2}(\pi(x, \xi))} \exp \left(-\int_{\pi(x, t)}^{\pi(x, \xi)} \frac{m(\tau)}{g(\tau)} d \tau\right) \\
& +u_{0}(\pi(x, t)) \frac{g^{\prime}(\pi(x, t))}{g(\pi(x, \xi))} \frac{g(\pi(x, t))}{g(\pi(x, \xi))} \exp \left(-\int_{\pi(x, t)}^{\pi(x, \xi)} \frac{m(\tau)}{g(\tau)} d \tau\right) \\
& -u_{0}(\pi(x, t)) \frac{g(\pi(x, t)) g^{\prime}(\pi(x, \xi))}{g^{2}(\pi(x, \xi))} \exp \left(-\int_{\pi(x, t)}^{\pi(x, \xi)} \frac{m(\tau)}{g(\tau)} d \tau\right) \\
& +u_{0}(\pi(x, t)) \frac{g(\pi(x, t))}{g(\pi(x, \xi))} \exp \left(-\int_{\pi(x, t)}^{\pi(x, \xi)} \frac{m(\tau)}{g(\tau)} d \tau\right)\left(\frac{m(\pi(x, t))}{g(\pi(x, \xi))}\right) \\
& -u_{0}(\pi(x, t)) \frac{g(\pi(x, t))}{g(\pi(x, \xi))} \exp \left(-\int_{\pi(x, t)}^{\pi(x, \xi)} \frac{m(\tau)}{g(\tau)} d \tau\right)\left(\frac{m(\pi(x, \xi))}{g(\pi(x, \xi))}\right)
\end{aligned}
$$

Let $\tau=G^{-1}(G(x)-\xi)$. Then we find that the following three equalities hold:

$$
\begin{aligned}
& \int_{0}^{t} \frac{h(\pi(x, \xi))}{g(\pi(x, \xi))} d \xi=\int_{\pi(x, t)}^{x} \frac{h(\tau)}{g^{2}(\tau)} d \tau, \\
& \int_{0}^{t} \frac{h(\pi(x, \xi)) g^{\prime}(\pi(x, \xi))}{g(\pi(x, \xi))} d \xi=\int_{\pi(x, t)}^{x} \frac{h(\tau) g^{\prime}(\tau)}{g^{2}(\tau)} d \tau, \\
& \int_{0}^{t} \frac{h(\pi(x, \xi)) m(\pi(x, \xi))}{g(\pi(x, \xi))} d \xi=\int_{\pi(x, t)}^{x} \frac{h(\tau) m(\tau)}{g^{2}(\tau)} d \tau .
\end{aligned}
$$


Using the above equalities and equation (78), we can rewrite the second term on the right side of (74) as

$$
\begin{aligned}
\int_{0}^{t} h(\pi(x, \xi)) u_{1}(\pi(x, \xi), t-\xi) \frac{g(\pi(x, \xi))}{g(x)} \exp \left(-\int_{\pi(x, \xi)}^{x} \frac{m(\tau)}{g(\tau)} d \tau\right) d \xi \\
=u_{0}^{\prime}(\pi(x, t)) \frac{g^{2}(\pi(x, t))}{g(x)} \exp \left(-\int_{\pi(x, t)}^{x} \frac{m(\tau)}{g(\tau)} d \tau\right)\left(\int_{\pi(x, t)}^{x} \frac{h(\tau)}{g^{2}(\tau)} d \tau\right) \\
+u_{0}(\pi(x, t)) \frac{g^{\prime}(\pi(x, t)) g(\pi(x, t))}{g(x)} \exp \left(-\int_{\pi(x, t)}^{x} \frac{m(\tau)}{g(\tau)} d \tau\right)\left(\int_{\pi(x, t)}^{x} \frac{h(\tau)}{g^{2}(\tau)} d \tau\right) \\
\quad-u_{0}(\pi(x, t)) \frac{g(\pi(x, t))}{g(x)} \exp \left(-\int_{\pi(x, t)}^{x} \frac{m(\tau)}{g(\tau)} d \tau\right)\left(\int_{\pi(x, t)}^{x} \frac{h(\tau) g^{\prime}(\tau)}{g^{2}(\tau)} d \tau\right) \\
+u_{0}(\pi(x, t)) \frac{g(\pi(x, t))}{g(x)} \exp \left(-\int_{\pi(x, t)}^{x} \frac{m(\tau)}{g(\tau)} d \tau\right)\left[m(\pi(x, t)) \int_{\pi(x, t)}^{x} \frac{h(\tau)}{g^{2}(\tau)} d \tau\right] \\
\quad-u_{0}(\pi(x, t)) \frac{g(\pi(x, t))}{g(x)} \exp \left(-\int_{\pi(x, t)}^{x} \frac{m(\tau)}{g(\tau)} d \tau\right)\left[\int_{\pi(x, t)}^{x} \frac{h(\tau) m(\tau)}{g^{2}(\tau)} d \tau\right] .
\end{aligned}
$$

Observe that

$$
\int_{\pi(x, t)}^{x} \frac{h(\tau) g^{\prime}(\tau)}{g^{2}(\tau)} d \tau-\int_{\pi(x, t)}^{x} \frac{h^{\prime}(\tau)}{g(\tau)} d \tau=\frac{h(\pi(x, t))}{g(\pi(x, t))}-\frac{h(x)}{g(x)} .
$$

Thus, by equations (74), (76) and (79), we find that for the case $x \geq G^{-1}(t)$,

$$
\begin{aligned}
e(x, t)= & -u_{0}^{\prime}(\pi(x, t)) \frac{g^{2}(\pi(x, t))}{g(x)} \exp \left(-\int_{\pi(x, t)}^{x} \frac{m(\tau)}{g(\tau)} d \tau\right)\left(\int_{\pi(x, t)}^{x} \frac{h(\tau)}{g^{2}(\tau)} d \tau\right) \\
& -u_{0}(\pi(x, t)) \frac{g^{\prime}(\pi(x, t)) g(\pi(x, t))}{g(x)} \exp \left(-\int_{\pi(x, t)}^{x} \frac{m(\tau)}{g(\tau)} d \tau\right)\left(\int_{\pi(x, t)}^{x} \frac{h(\tau)}{g^{2}(\tau)} d \tau\right) \\
& +u_{0}(\pi(x, t)) \frac{g(\pi(x, t))}{g(x)} \exp \left(-\int_{\pi(x, t)}^{x} \frac{m(\tau)}{g(\tau)} d \tau\right)\left(\frac{h(\pi(x, t))}{g(\pi(x, t))}-\frac{h(x)}{g(x)}\right) \\
& -u_{0}(\pi(x, t)) \frac{g(\pi(x, t))}{g(x)} \exp \left(-\int_{\pi(x, t)}^{x} \frac{m(\tau)}{g(\tau)} d \tau\right)\left[m(\pi(x, t)) \int_{\pi(x, t)}^{x} \frac{h(\tau)}{g^{2}(\tau)} d \tau\right] \\
& +u_{0}(\pi(x, t)) \frac{g(\pi(x, t))}{g(x)} \exp \left(-\int_{\pi(x, t)}^{x} \frac{m(\tau)}{g(\tau)} d \tau\right)\left[\int_{\pi(x, t)}^{x} \frac{h(\tau) m(\tau)}{g^{2}(\tau)} d \tau\right] .
\end{aligned}
$$

Thus, equations (67) and (80) imply that

$$
u_{g}(x, t ; g, h)=e(x, t) \text {, if } x \geq G^{-1}(t) .
$$


We next simplify the expression in (75). Let $\tau=G^{-1}(t-\xi)$. Then we find that

$$
\int_{t-G(x)}^{t} h^{\prime}\left(G^{-1}(t-\xi)\right) d \xi=\int_{0}^{x} \frac{h^{\prime}(\tau)}{g(\tau)} d \tau=\frac{h(x)}{g(x)}-\frac{h(0)}{g(0)}+\int_{0}^{x} \frac{h(\tau)}{g^{2}(\tau)} g^{\prime}(\tau) d \tau .
$$

Thus, using (45) and this equality, we can rewrite the second term on the right side of (75) as

$$
\begin{gathered}
\int_{t-G(x)}^{t} h^{\prime}(\varrho(t, \xi)) u(\varrho(t, \xi), \rho(x, t, \xi)) \frac{g(\varrho(t, \xi))}{g(x)} \exp \left(-\int_{\varrho(t, \xi)}^{x} \frac{m(\tau)}{g(\tau)} d \tau\right) d \xi \\
=\frac{B(t-G(x))}{g(x)} \exp \left(-\int_{0}^{x} \frac{m(\tau)}{g(\tau)} d \tau\right)\left[\frac{h(x)}{g(x)}-\frac{h(0)}{g(0)}+\int_{0}^{x} \frac{h(\tau)}{g^{2}(\tau)} g^{\prime}(\tau) d \tau\right] .
\end{gathered}
$$

The continuous differentiability of $B$ and assumptions (A1) and (A2) imply that $u$ is continuously differentiable in the region $\left\{(x, t) \mid 0 \leq x<G^{-1}(t), t>0\right\}$, and $u_{x}$ is given by

$$
\begin{aligned}
u_{x}(x, t)= & -\frac{B^{\prime}(t-G(x))}{g^{2}(x)} \exp \left(-\int_{0}^{x} \frac{m(\xi)}{g(\xi)} d \xi\right) \\
& -\frac{B(t-G(x))}{g^{2}(x)} g^{\prime}(x) \exp \left(-\int_{0}^{x} \frac{m(\xi)}{g(\xi)} d \xi\right) \\
& -\frac{B(t-G(x))}{g(x)} \frac{m(x)}{g(x)} \exp \left(-\int_{0}^{x} \frac{m(\xi)}{g(\xi)} d \xi\right) .
\end{aligned}
$$

Observe that $\rho(x, t, \xi)-G(\varrho(x, \xi))=t-G(x)$. Hence, we find that

$$
\begin{aligned}
u_{1}(\varrho(x, \xi), \rho(x, t, \xi))= & -\frac{B^{\prime}(t-G(x))}{g^{2}(\varrho(x, \xi))} \exp \left(-\int_{0}^{\varrho(x, \xi)} \frac{m(\tau)}{g(\tau)} d \tau\right) \\
& -\frac{B(t-G(x))}{g^{2}(\varrho(x, \xi))} g^{\prime}(\varrho(x, \xi)) \exp \left(-\int_{0}^{\varrho(x, \xi)} \frac{m(\tau)}{g(\tau)} d \tau\right) \\
& -\frac{B(t-G(x))}{g(\varrho(x, \xi))} \frac{m(\varrho(x, \xi))}{g(\varrho(x, \xi))} \exp \left(-\int_{0}^{\varrho(x, \xi)} \frac{m(\tau)}{g(\tau)} d \tau\right) .
\end{aligned}
$$


Let $\tau=G^{-1}(t-\xi)$. Then $d \xi=-\frac{1}{g(\tau)} d \tau$. Hence, the following three equalities hold:

$$
\begin{aligned}
& \int_{t-G(x)}^{t} \frac{h(\varrho(t, \xi))}{g(\varrho(t, \xi))} d \xi=\int_{0}^{x} \frac{h(\tau)}{g^{2}(\tau)} d \tau \\
& \int_{t-G(x)}^{t} \frac{h(\varrho(t, \xi)) g^{\prime}(\varrho(t, \xi))}{g(\varrho(t, \xi))} d \xi=\int_{0}^{x} \frac{h(\tau)}{g^{2}(\tau)} g^{\prime}(\tau) d \tau \\
& \int_{t-G(x)}^{t} \frac{h(\varrho(t, \xi)) m(\varrho(t, \xi))}{g(\varrho(t, \xi))} d \xi=\int_{0}^{x} \frac{h(\tau) m(\tau)}{g^{2}(\tau)} d \tau .
\end{aligned}
$$

Thus, by the above three equalities, the third term on the right side of (75) can be rewritten as

$$
\begin{array}{rl}
\int_{t-G(x)}^{t} & h(\varrho(t, \xi)) u_{1}(\varrho(t, \xi), \rho(x, t, \xi)) \frac{g(\varrho(t, \xi))}{g(x)} \exp \left(-\int_{\varrho(t, \xi)}^{x} \frac{m(\tau)}{g(\tau)} d \tau\right) d \xi \\
= & -\frac{B^{\prime}(t-G(x))}{g(x)} \exp \left(-\int_{0}^{x} \frac{m(\tau)}{g(\tau)} d \tau\right)\left(\int_{0}^{x} \frac{h(\tau)}{g^{2}(\tau)} d \tau\right) \\
& -\frac{B(t-G(x))}{g(x)} \exp \left(-\int_{0}^{x} \frac{m(\tau)}{g(\tau)} d \tau\right)\left(\int_{0}^{x} \frac{h(\tau)}{g^{2}(\tau)} g^{\prime}(\tau) d \tau\right) \\
& -\frac{B(t-G(x))}{g(x)} \exp \left(-\int_{0}^{x} \frac{m(\tau)}{g(\tau)} d \tau\right)\left(\int_{0}^{x} \frac{h(\tau) m(\tau)}{g^{2}(\tau)} d \tau\right) .
\end{array}
$$

Therefore, from equations (175), (82) and (85), we obtain that if $x<G^{-1}(t)$, then

$$
\begin{aligned}
e(x, t)= & \frac{E(t-G(x))}{g(x)} \exp \left(-\int_{0}^{x} \frac{m(\tau)}{g(\tau)} d \tau\right) \\
& +\frac{B^{\prime}(t-G(x))}{g(x)} \exp \left(-\int_{0}^{x} \frac{m(\tau)}{g(\tau)} d \tau\right)\left(\int_{0}^{x} \frac{h(\tau)}{g^{2}(\tau)} d \tau\right) \\
& -\frac{B(t-G(x))}{g^{2}(x)} h(x) \exp \left(-\int_{0}^{x} \frac{m(\tau)}{g(\tau)} d \tau\right) \\
& +\frac{B(t-G(x))}{g(x)} \exp \left(-\int_{0}^{x} \frac{m(\tau)}{g(\tau)} d \tau\right)\left(\int_{0}^{x} \frac{h(\tau) m(\tau)}{g^{2}(\tau)} d \tau\right) .
\end{aligned}
$$

From equations (73) and (86)), we see that if we want to show that $u_{g}(x, t ; g, h)=e(x, t)$ for the case $x<G^{-1}(t)$, then we need to show that $E(t)=B_{g}(t ; g, h)$ for $t \in[0, T]$. Let 
$x=G^{-1}(\eta)$. Then $\eta=G(x)$ and $d \eta=\frac{1}{g(x)} d x$. Hence, we have

$$
\int_{0}^{t} k_{g}(\eta ; g, h) B(t-\eta) d \eta=\int_{0}^{G^{-1}(t)} k_{g}(G(x) ; g, h) \frac{B(t-G(x))}{g(x)} d x .
$$

Let $\xi=G^{-1}(\sigma)$ so that $d \sigma=\frac{1}{g(\xi)} d \xi$. Hence, we have

$$
\exp \left(-\int_{0}^{G(x)} m\left(G^{-1}(\sigma)\right) d \sigma\right)=\exp \left(-\int_{0}^{x} \frac{m(\xi)}{g(\xi)} d \xi\right)
$$

and

$$
\begin{aligned}
\int_{0}^{G(x)} & m^{\prime}\left(G^{-1}(\sigma)\right) g\left(G^{-1}(\sigma)\right)\left(\int_{0}^{G^{-1}(\sigma)} \frac{h(\tau)}{g^{2}(\tau)} d \tau\right) d \sigma \\
= & \int_{0}^{x} m^{\prime}(\xi)\left(\int_{0}^{\xi} \frac{h(\tau)}{g^{2}(\tau)} d \tau\right) d \xi \\
= & m(x) \int_{0}^{x} \frac{h(\xi)}{g^{2}(\xi)} d \xi-\int_{0}^{x} \frac{h(\xi) m(\xi)}{g^{2}(\xi)} d \xi
\end{aligned}
$$

Hence, by equations (71), (88) and (89), we find that

$$
\begin{aligned}
k_{g}(G(x) ; g, h)= & \beta^{\prime}(x) g(x)\left(\int_{0}^{x} \frac{h(\xi)}{g^{2}(\xi)} d \xi\right) \exp \left(-\int_{0}^{x} \frac{m(\xi)}{g(\xi)} d \xi\right) \\
& -\beta(x) m(x)\left(\int_{0}^{x} \frac{h(\xi)}{g^{2}(\xi)} d \xi\right) \exp \left(-\int_{0}^{x} \frac{m(\xi)}{g(\xi)} d \xi\right) \\
& +\beta(x) \exp \left(-\int_{0}^{x} \frac{m(\xi)}{g(\xi)} d \xi\right)\left(\int_{0}^{x} \frac{h(\xi) m(\xi)}{g^{2}(\xi)} d \xi\right) .
\end{aligned}
$$

From equations (87) and (90), we obtain that

$$
\begin{aligned}
\int_{0}^{t} k_{g}( & ; g, h) B(t-\eta) d \eta \\
= & \int_{0}^{G^{-1}(t)} \beta^{\prime}(x) B(t-G(x))\left(\int_{0}^{x} \frac{h(\xi)}{g^{2}(\xi)} d \xi\right) \exp \left(-\int_{0}^{x} \frac{m(\xi)}{g(\xi)} d \xi\right) d x \\
& -\int_{0}^{G^{-1}(t)} \beta(x) \frac{B(t-G(x))}{g(x)} m(x)\left(\int_{0}^{x} \frac{h(\xi)}{g^{2}(\xi)} d \xi\right) \exp \left(-\int_{0}^{x} \frac{m(\xi)}{g(\xi)} d \xi\right) d x \\
& +\int_{0}^{G^{-1}(t)} \beta(x) \frac{B(t-G(x))}{g(x)} \exp \left(-\int_{0}^{x} \frac{m(\xi)}{g(\xi)} d \xi\right)\left(\int_{0}^{x} \frac{h(\xi) m(\xi)}{g^{2}(\xi)} d \xi\right) d x .
\end{aligned}
$$


Next observe that the first term on the right side of (91) can be rewritten as

$$
\begin{aligned}
\int_{0}^{G^{-1}(t)} & \beta^{\prime}(x) B(t-G(x))\left(\int_{0}^{x} \frac{h(\xi)}{g^{2}(\xi)} d \xi\right) \exp \left(-\int_{0}^{x} \frac{m(\xi)}{g(\xi)} d \xi\right) d x \\
= & \beta\left(G^{-1}(t)\right) B(0)\left(\int_{0}^{G^{-1}(t)} \frac{h(\xi)}{g^{2}(\xi)} d \xi\right) \exp \left(-\int_{0}^{G^{-1}(t)} \frac{m(\xi)}{g(\xi)} d \xi\right) \\
& +\int_{0}^{G^{-1}(t)} \beta(x) \frac{B^{\prime}(t-G(x))}{g(x)} \exp \left(-\int_{0}^{x} \frac{m(\xi)}{g(\xi)} d \xi\right)\left(\int_{0}^{x} \frac{h(\xi)}{g^{2}(\xi)} d \xi\right) d x \\
& +\int_{0}^{G^{-1}(t)} \beta(x) \frac{B(t-G(x))}{g(x)} m(x) \exp \left(-\int_{0}^{x} \frac{m(\xi)}{g(\xi)} d \xi\right)\left(\int_{0}^{x} \frac{h(\xi)}{g^{2}(\xi)} d \xi\right) d x \\
& -\int_{0}^{G^{-1}(t)} \beta(x) \frac{B(t-G(x))}{g^{2}(x)} h(x) \exp \left(-\int_{0}^{x} \frac{m(\xi)}{g(\xi)} d \xi\right) d x .
\end{aligned}
$$

Hence, from equations (91) and (92) we observe that

$$
\begin{aligned}
\int_{0}^{t} k_{g}(\eta ; g, h) B(t-\eta) d \eta \\
=\beta\left(G^{-1}(t)\right) B(0)\left(\int_{0}^{G^{-1}(t)} \frac{h(\xi)}{g^{2}(\xi)} d \xi\right) \exp \left(-\int_{0}^{G^{-1}(t)} \frac{m(\xi)}{g(\xi)} d \xi\right) \\
\quad+\int_{0}^{G^{-1}(t)} \beta(x) \frac{B^{\prime}(t-G(x))}{g(x)} \exp \left(-\int_{0}^{x} \frac{m(\xi)}{g(\xi)} d \xi\right)\left(\int_{0}^{x} \frac{h(\xi)}{g^{2}(\xi)} d \xi\right) d x \\
\quad-\int_{0}^{G^{-1}(t)} \beta(x) \frac{B(t-G(x))}{g^{2}(x)} h(x) \exp \left(-\int_{0}^{x} \frac{m(\xi)}{g(\xi)} d \xi\right) d x \\
\quad+\int_{0}^{G^{-1}(t)} \beta(x) \frac{B(t-G(x))}{g(x)} \exp \left(-\int_{0}^{x} \frac{m(\xi)}{g(\xi)} d \xi\right)\left(\int_{0}^{x} \frac{h(\xi) m(\xi)}{g^{2}(\xi)} d \xi\right) d x .
\end{aligned}
$$

We return to (69) to simplify the expression for $F_{g}(t ; g, h)$. Let $\xi=G^{-1}(G(x)-t+\sigma)$. Then $d \sigma=\frac{1}{g(\xi)} d \xi$. Hence, we find that

$$
\exp \left(-\int_{0}^{t} m\left(G^{-1}(G(x)-t+\sigma)\right) d \sigma\right)=\exp \left(-\int_{\pi(x, t)}^{x} \frac{m(\xi)}{g(\xi)} d \xi\right) .
$$


Let $x=G^{-1}(G(\eta)+t)$. Then $\eta=\pi(x, t)$ and $\pi(\eta,-\sigma)=G^{-1}(G(x)-t+\sigma)$. Hence, using this transformation, equations (94) and (62), and the facts that $\pi(\pi(x, t),-t)=x$ and $\pi\left(G^{-1}(t), t\right)=0$, we can rewrite the first term on the right side of (69) as follows:

$$
\begin{aligned}
\int_{0}^{\bar{x}} u_{0}(\eta) \beta^{\prime}(\pi(\eta,-t)) g(\pi(\eta,-t))\left(\int_{\eta}^{\pi(\eta,-t)} \frac{h(\tau)}{g^{2}(\tau)} d \tau\right) \exp \left(-\int_{0}^{t} m(\pi(\eta,-\sigma)) d \sigma\right) d \eta \\
=\int_{G^{-1}(t)}^{\bar{x}} \beta^{\prime}(x) u_{0}(\pi(x, t)) g(\pi(x, t))\left(\int_{\pi(x, t)}^{x} \frac{h(\xi)}{g^{2}(\xi)} d \xi\right) \exp \left(-\int_{\pi(x, t)}^{x} \frac{m(\xi)}{g(\xi)} d \xi\right) d x \\
=-\beta\left(G^{-1}(t)\right) u_{0}(0) g(0)\left(\int_{0}^{G^{-1}(t)} \frac{h(\xi)}{g^{2}(\xi)} d \xi\right) \exp \left(-\int_{0}^{G^{-1}(t)} \frac{m(\xi)}{g(\xi)} d \xi\right) \\
-\int_{G^{-1}(t)}^{\bar{x}} \beta(x) u_{0}^{\prime}(\pi(x, t)) \frac{g^{2}(\pi(x, t))}{g(x)}\left(\int_{\pi(x, t)}^{x} \frac{h(\xi)}{g^{2}(\xi)} d \xi\right) \exp \left(-\int_{\pi(x, t)}^{x} \frac{m(\xi)}{g(\xi)} d \xi\right) d x \\
-\int_{G^{-1}(t)}^{\bar{x}} \beta(x) u_{0}(\pi(x, t)) g^{\prime}(\pi(x, t)) \frac{g(\pi(x, t))}{g(x)}\left(\int_{\pi(x, t)}^{x} \frac{h(\xi)}{g^{2}(\xi)} d \xi\right) \\
\quad \times \exp \left(-\int_{\pi(x, t)}^{x} \frac{m(\xi)}{g(\xi)} d \xi\right) d x \\
-\int_{G^{-1}(t)}^{\bar{x}} \beta(x) u_{0}(\pi(x, t)) g(\pi(x, t))\left[\frac{h(x)}{g^{2}(x)}-\frac{h(\pi(x, t))}{g(x) g(\pi(x, t))}\right] \\
\quad \times \exp \left(-\int_{\pi(x, t)}^{x} \frac{m(\xi)}{g(\xi)} d \xi\right) d x \\
+\int_{G^{-1}(t)}^{\bar{x}} \beta(x) u_{0}(\pi(x, t)) g(\pi(x, t))\left(\int_{\pi(x, t)}^{x} \frac{h(\xi)}{g^{2}(\xi)} d \xi\right) \\
\quad \times \exp \left(-\int_{\pi(x, t)}^{x} \frac{m(\xi)}{g(\xi)} d \xi\right) \frac{m(x)}{g(x)} d x \\
-\int_{G^{-1}(t)}^{\bar{x}} \beta(x) u_{0}(\pi(x, t)) g(\pi(x, t))\left(\int_{\pi(x, t)}^{x} \frac{h(\xi)}{g^{2}(\xi)} d \xi\right) \\
\quad \times \exp \left(-\int_{\pi(x, t)}^{x} \frac{m(\xi)}{g(\xi)} d \xi\right) \frac{m(\pi(x, t))}{g(x)} d x \\
\quad \\
\quad \\
\quad
\end{aligned}
$$

By the same transformation as we derived for (94), we obtain that

$$
\begin{aligned}
& \int_{0}^{t} m^{\prime}\left(G^{-1}(G(x)-t+\sigma)\right) g\left(G^{-1}(G(x)-t+\sigma)\right)\left(\int_{\pi(x, t)}^{G^{-1}(G(x)-t+\sigma)} \frac{h(\tau)}{g^{2}(\tau)} d \tau\right) d \sigma \\
& \quad=\int_{\pi(x, t)}^{x} m^{\prime}(\xi)\left(\int_{\pi(x, t)}^{\xi} \frac{h(\tau)}{g^{2}(\tau)} d \tau\right) d \xi \\
& \quad=m(x) \int_{\pi(x, t)}^{x} \frac{h(\tau)}{g^{2}(\tau)} d \tau-\int_{\pi(x, t)}^{x} \frac{h(\xi) m(\xi)}{g^{2}(\xi)} d \xi .
\end{aligned}
$$


Hence, by equations (96), (94) and (68), the second term on the right side of (69) can be rewritten as

$$
\begin{aligned}
& \int_{0}^{\bar{x}} u_{0}(\eta) \beta(\pi(\eta,-t)) \exp \left(-\int_{0}^{t} m(\pi(\eta,-\sigma)) d \sigma\right)\left[\int_{0}^{t} m^{\prime}(\pi(\eta,-\sigma)) \pi_{g}(\eta,-\sigma ; g, h) d \sigma\right] d \eta \\
& =\int_{G^{-1}(t)}^{\bar{x}} \beta(x) u_{0}(\pi(x, t)) \frac{g(\pi(x, t))}{g(x)} \exp \left(-\int_{\pi(x, t)}^{x} \frac{m(\xi)}{g(\xi)} d \xi\right)\left[m(x) \int_{\pi(x, t)}^{x} \frac{h(\tau)}{g^{2}(\tau)} d \tau\right] d x \\
& -\int_{G^{-1}(t)}^{\bar{x}} \beta(x) u_{0}(\pi(x, t)) \frac{g(\pi(x, t))}{g(x)} \exp \left(-\int_{\pi(x, t)}^{x} \frac{m(\xi)}{g(\xi)} d \xi\right)\left(\int_{\pi(x, t)}^{x} \frac{h(\xi) m(\xi)}{g^{2}(\xi)} d \xi\right) d x
\end{aligned}
$$

Thus, equations (69), (97) and (95) imply that

$$
\begin{aligned}
& F_{g}(t ; g, h)=-\beta\left(G^{-1}(t)\right) u_{0}(0) g(0)\left(\int_{0}^{G^{-1}(t)} \frac{h(\xi)}{g^{2}(\xi)} d \xi\right) \exp \left(-\int_{0}^{G^{-1}(t)} \frac{m(\xi)}{g(\xi)} d \xi\right) \\
& -\int_{G^{-1}(t)}^{\bar{x}} \beta(x) u_{0}^{\prime}(\pi(x, t)) \frac{g^{2}(\pi(x, t))}{g(x)}\left(\int_{\pi(x, t)}^{x} \frac{h(\xi)}{g^{2}(\xi)} d \xi\right) \\
& \times \exp \left(-\int_{\pi(x, t)}^{x} \frac{m(\xi)}{g(\xi)} d \xi\right) d x \\
& -\int_{G^{-1}(t)}^{\bar{x}} \beta(x) u_{0}(\pi(x, t)) g^{\prime}(\pi(x, t)) \frac{g(\pi(x, t))}{g(x)}\left(\int_{\pi(x, t)}^{x} \frac{h(\xi)}{g^{2}(\xi)} d \xi\right) \\
& \times \exp \left(-\int_{\pi(x, t)}^{x} \frac{m(\xi)}{g(\xi)} d \xi\right) d x \\
& -\int_{G^{-1}(t)}^{\bar{x}} \beta(x) u_{0}(\pi(x, t)) g(\pi(x, t))\left[\frac{h(x)}{g^{2}(x)}-\frac{h(\pi(x, t))}{g(x) g(\pi(x, t))}\right] \\
& \times \exp \left(-\int_{\pi(x, t)}^{x} \frac{m(\xi)}{g(\xi)} d \xi\right) d x \\
& -\int_{G^{-1}(t)}^{\bar{x}} \beta(x) u_{0}(\pi(x, t)) g(\pi(x, t))\left(\int_{\pi(x, t)}^{x} \frac{h(\xi)}{g^{2}(\xi)} d \xi\right) \\
& \times \exp \left(-\int_{\pi(x, t)}^{x} \frac{m(\xi)}{g(\xi)} d \xi\right) \frac{m(\pi(x, t))}{g(x)} d x \\
& +\int_{G^{-1}(t)}^{\bar{x}} \beta(x) u_{0}(\pi(x, t)) \frac{g(\pi(x, t))}{g(x)} \\
& \times \exp \left(-\int_{\pi(x, t)}^{x} \frac{m(\xi)}{g(\xi)} d \xi\right)\left(\int_{\pi(x, t)}^{x} \frac{h(\xi) m(\xi)}{g^{2}(\xi)} d \xi\right) d x .
\end{aligned}
$$

Let $\eta=G(x)$. Then $d \eta=\frac{1}{g(x)} d x$. Hence we find that

$$
\int_{0}^{G^{-1}(t)} \beta(x) \frac{E(t-G(x))}{g(x)} \exp \left(-\int_{0}^{x} \frac{m(\tau)}{g(\tau)} d \tau\right) d x=\int_{0}^{t} k(\eta) E(t-\eta) d \eta
$$


Observe that assumption (A5) implies that $B(0)=g(0) u_{0}(0)$. Hence, from equations (80), (86), (93), (98), we see that

$$
E(t)=\int_{0}^{t} k(\eta) E(t-\eta) d \eta+\int_{0}^{t} k_{g}(\eta ; g, h) B(t-\eta) d \eta+F_{g}(t ; g, h)
$$

Using equations (72) and (100), and the uniqueness of the solution to (72), we have that

$$
E(t)=B_{g}(t ; g, h) \text { for any } t \in[0, T]
$$

Hence, equations (73), (86) and (101) imply that

$$
u_{g}(x, t ; g, h)=e(x, t) \text {, if } x<G^{-1}(t) .
$$

Thus, from (81) and (102) we know that $u_{g}(x, t ; g, h)$ satisfies the initial boundary value problem (59), which means that we can use this system to solve for $u_{g}(x, t ; g, h)$.

4. Numerical results. We illustrate the ease in computation of the sensitivities derived in Section 3 with a simple example. In partiular, we consider the computation of the sensitivities of $u$ with respect to $m$ and $g$, and we use an implicit finite difference scheme to approximate the population density $u(x, t)$ as well as $w(x, t)=u_{m}(x, t ; m, h)$ and $e(x, t)=u_{g}(x, t ; g, h)$. Let $\Delta x=\bar{x} / n$ and $\Delta t=T / l$ be the spatial and time mesh sizes, respectively. The mesh points are given by $x_{j}=j \Delta x, j=0,1,2, \ldots, n$, and $t_{k}=$ $k \Delta t, k=0,1,2, \ldots, l$. We denote by $u_{j}^{k}$ the finite difference approximation of $u\left(x_{j}, t_{k}\right)$, and we let $u_{j}^{0}=u_{0}\left(x_{j}\right), g_{j}=g\left(x_{j}\right), m_{j}=m\left(x_{j}\right)$, and $\beta_{j}=\beta\left(x_{j}\right), j=0,1,2, \ldots, n$. The finite difference scheme used to approximate the solution $u(x, t)$ to (1) is given as

$$
\begin{aligned}
& \frac{u_{j}^{k+1}-u_{j}^{k}}{\Delta t}+\frac{g_{j} u_{j}^{k+1}-g_{j-1} u_{j-1}^{k+1}}{\Delta x}+m_{j} u_{j}^{k+1}=0, \quad 1 \leq j \leq n, \\
& g_{0} u_{0}^{k+1}=\sum_{i=1}^{n} \beta_{i} u_{i}^{k} \Delta x .
\end{aligned}
$$

We denote by $w_{j}^{k}$ the finite difference approximation of $w\left(x_{j}, t_{k}\right)$, and we let $w_{j}^{0}=0$ and $h_{j}=h\left(x_{j}\right)$. The scheme used to approximate the solution $w(x, t)$ to (40) is given by

$$
\begin{aligned}
& \frac{w_{j}^{k+1}-w_{j}^{k}}{\Delta t}+\frac{g_{j} w_{j}^{k+1}-g_{j-1} w_{j-1}^{k+1}}{\Delta x}+m_{j} w_{j}^{k+1}+h_{j} u_{j}^{k+1}=0, \quad 1 \leq j \leq n, \\
& g_{0} w_{0}^{k+1}=\sum_{i=1}^{n} \beta_{i} w_{i}^{k} \Delta x .
\end{aligned}
$$


Let $e_{j}^{k}$ represent the finite difference approximation of $e\left(x_{j}, t_{k}\right)$, and $e_{j}^{0}=0, j=$ $0,1,2, \ldots, n$. Then the scheme used to approximate the solution $e(x, t)$ to (59) is given by

$$
\begin{aligned}
& \frac{e_{j}^{k+1}-e_{j}^{k}}{\Delta t}+\frac{g_{j} e_{j}^{k+1}-g_{j-1} e_{j-1}^{k+1}}{\Delta x}+m_{j} e_{j}^{k+1}+\frac{h_{j} u_{j}^{k+1}-h_{j-1} u_{j-1}^{k+1}}{\Delta x}=0, \quad 1 \leq j \leq n, \\
& g_{0} e_{0}^{k+1}+h_{0} u_{0}^{k+1}=\sum_{i=1}^{n} \beta_{i} e_{i}^{k} \Delta x .
\end{aligned}
$$

In this example, we assume that the maximum size $\bar{x}=1$ and the maximum time period $T=5$. The initial condition and model parameters are given as follows:

$$
u^{0}(x)=10 x^{3}(1-x), \quad g(x)=0.2(1-x), \quad m(x)=0.4, \quad \beta(x)=0 .
$$

Then by (11) and (12) we can solve analytically for $u(x, t)$ :

$$
u(x, t)= \begin{cases}10(1-x)\left[1-(1-x) e^{0.2 t}\right]^{3} & x \geq 1-e^{-0.2 t} \\ 0 & x<1-e^{-0.2 t}\end{cases}
$$

Note that $m$ is a constant, and hence we allow the direction $h=1$ when we take the directional derivative of $u$ with respect to $m$. Then by (50) and (52) we can solve analytically for $w(x, t)$ :

$$
w(x, t)= \begin{cases}-10 t(1-x)\left[1-(1-x) e^{0.2 t}\right]^{3} & x \geq 1-e^{-0.2 t} \\ 0 & x<1-e^{-0.2 t}\end{cases}
$$

In our example for $u_{g}$, the direction $h$ is chosen to be $h=2 g$ in taking the directional derivative of $u$ with respect to $g$. By (80) and (86) we can solve analytically for $e(x, t)$ :

$$
e(x, t)= \begin{cases}4 t(1-x)\left[1-(1-x) e^{0.2 t}\right]^{2}\left[5\left(1-(1-x) e^{0.2 t}\right)-3\right] & x \geq 1-e^{-0.2 t} \\ 0 & x<1-e^{-0.2 t}\end{cases}
$$

In Figures 11 and 2, the approximate solutions with $\Delta x=10^{-4}$ and $\Delta t=10^{-3}$ are depicted. The analytical solution, the approximate solution and the difference between them for $u(x, t)$ are plotted in Figure 1, those for $w(x, t)$ and $e(x, t)$ are plotted in Figure 2. These figures demonstrate that numerical solutions for $u(x, t), w(x, t)$ and $e(x, t)$ provide reasonable approximations to the analytical solutions. However, we also observe that the absolute value of the error becomes larger as time evolves. The reason for this is that the population distribution becomes narrower and steeper (see Figures 3 15) as time progresses because $g^{\prime}=-0.2<0$ in this example; this provides computational challenges. 

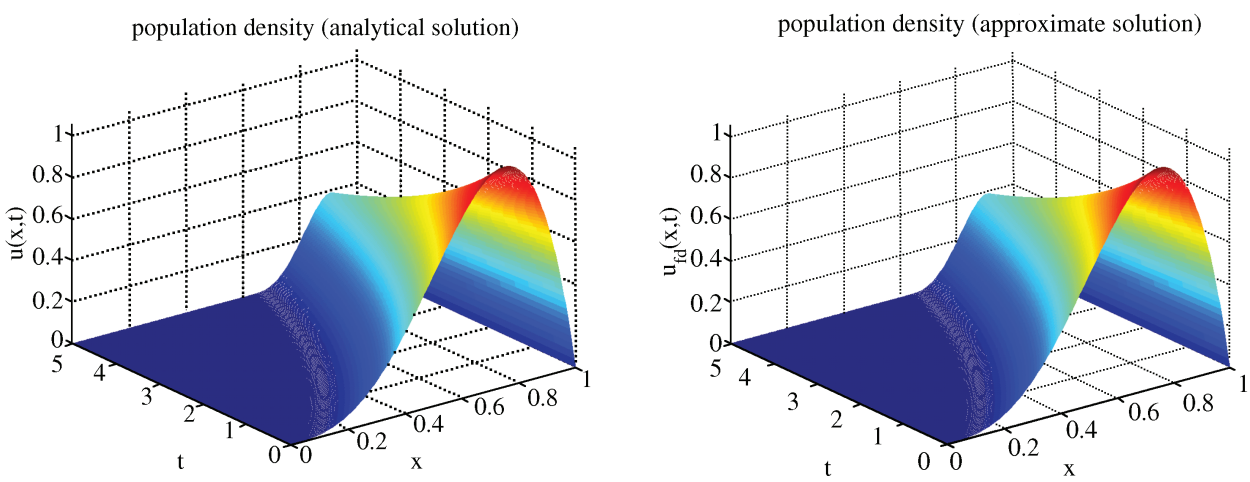

error between analytical solution and approximate solution: $\mathrm{u}(\mathrm{x}, \mathrm{t})$

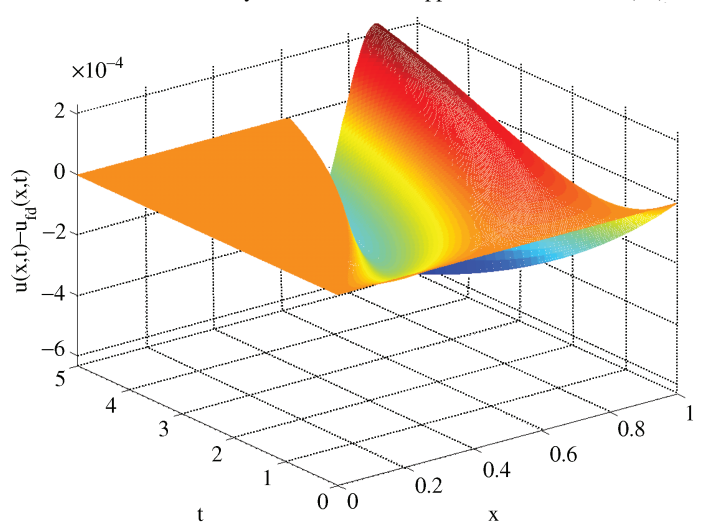

Fig. 1. (top left): Analytical solution for $u(x, t)$. (top right): The finite difference approximation $u_{f d}(x, t)$ of $u(x, t)$. (bottom): Error between the analytical solution and the finite difference approximation.

5. Concluding remarks. In this paper, we have considered the widely used SinkoStreifer/McKendrick-von Foerster equations for size-structured populations. We addressed the challenging problem of a rigorous derivation of the partial differential equations for the sensitivities of the population density with respect to initial conditions $u_{0}$, growth $g$, mortality $m$ and fecundity $\beta$. We used the method of characteristics to carry out the sensitivity analysis for the linear size-structured population model. This approach is tedious but very straightforward, and we believe it can be applied to a diverse class of more complicated structured population and biomass production models such as the size and class age structured epidemic model in [7]. A reassuring aspect of our investigations is that they reveal that the correct sensitivity equations can be formally obtained by simply differentiating the population equation of interest with respect to the functions $u_{0}, g, m$ and $\beta$. 


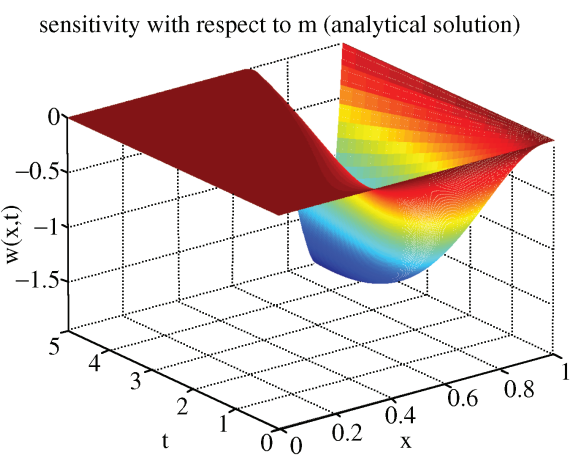

sensitivity with respect to m(approximate solution)

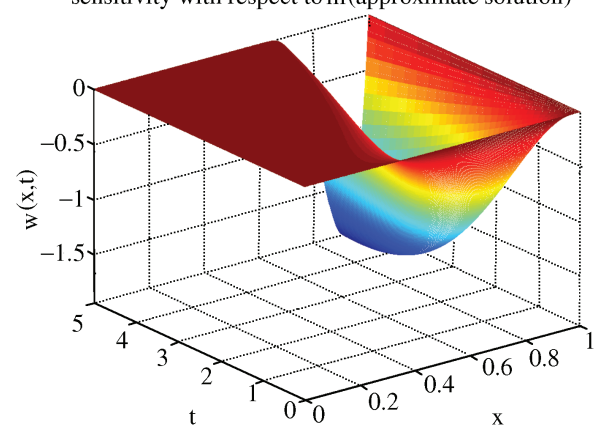

error between analytical solution and approximate solution: $\mathrm{w}(\mathrm{x}, \mathrm{t})$

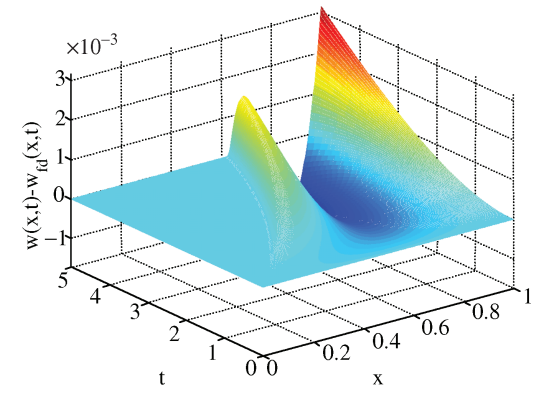

sensitivity with respect to $g$ (analytical solution)

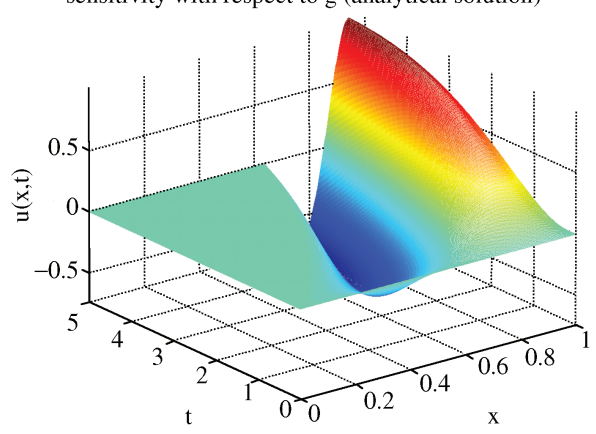

sensitivity with respect to $\mathrm{g}$ (approximate solution)

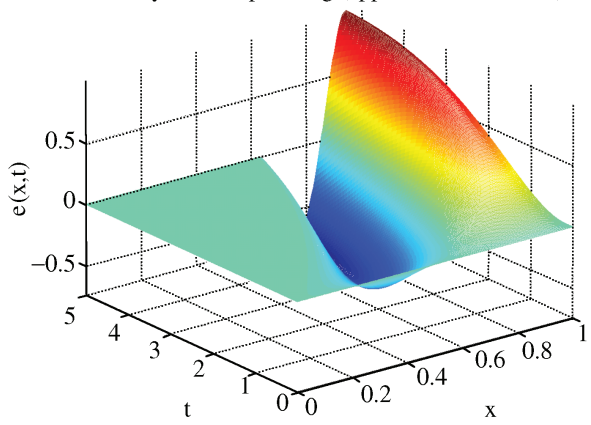

error between analytical solution and approximate solution: $\mathrm{e}(\mathrm{x}, \mathrm{t})$

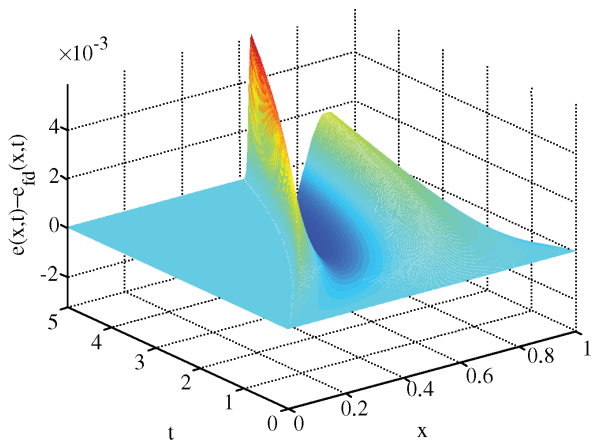

Fig. 2. (left column): Analytical solution, approximate solution and error between them for $w(x, t)$. (right column): Analytical solution, approximate solution and error between them for $e(x, t)$.

Acknowledgements. This research was supported in part by the National Institute of Allergy and Infectious Disease under grant 9R01AI071915-05, in part by the U.S. Air Force Office of Scientific Research under grant AFOSR-FA9550-04-1-0220 and in part by the Defense Advanced Research Projects Agency and Advanced BioNutrition Corp under grant ABNDTRA-0507-03. 

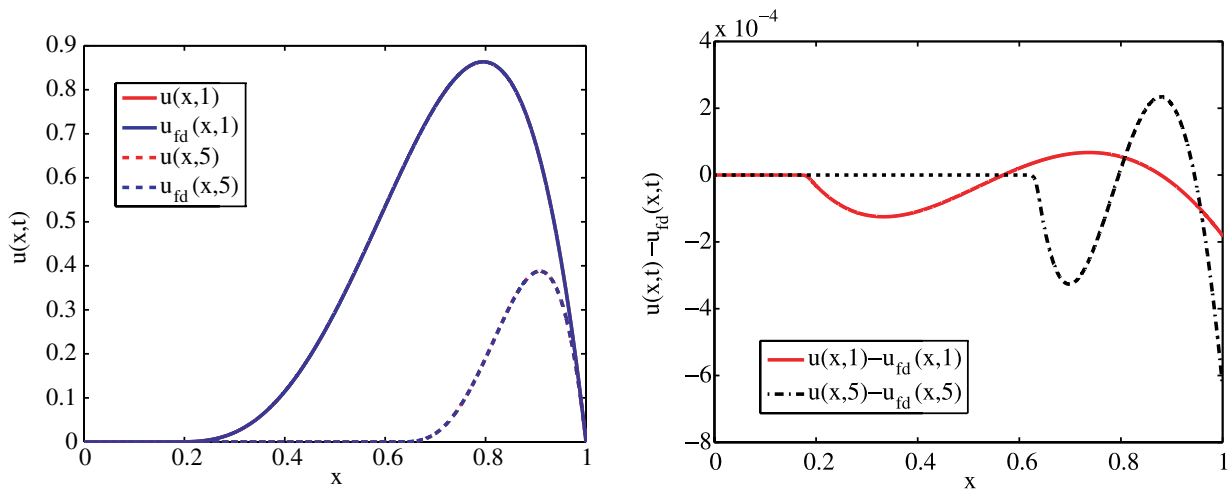

Fig. 3. (left): Analytical solution and approximate solution for population density at $t=1$ and $t=5$. (right): Error between analytical solution and approximate solution at $t=1$ and $t=5$.
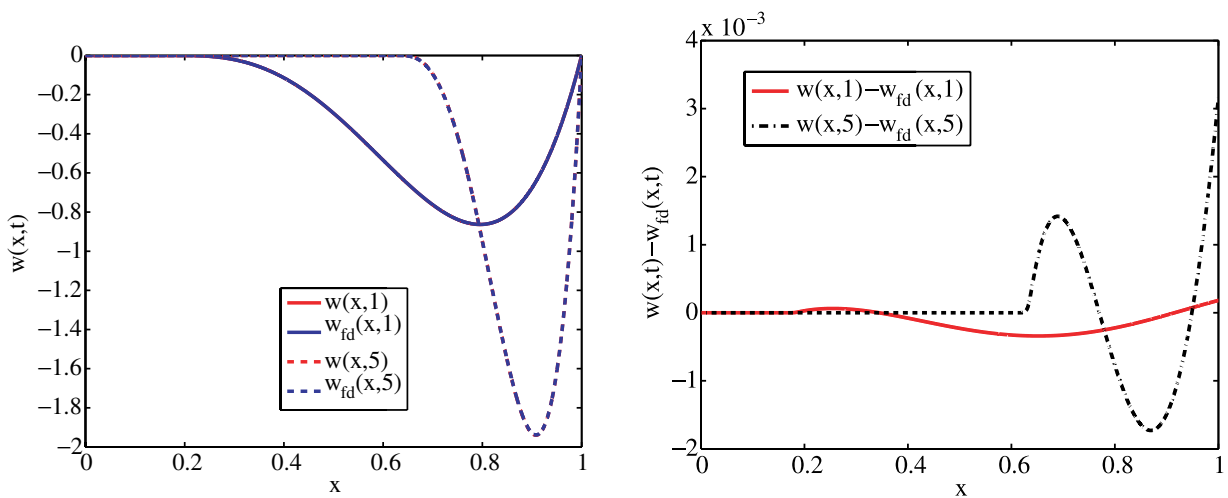

FIG. 4. (left): Analytical solution and approximate solution for sensitivity with respect to $m$ at $t=1$ and $t=5$. (right): Error between analytical solution and approximate solution at $t=1$ and $t=5$.
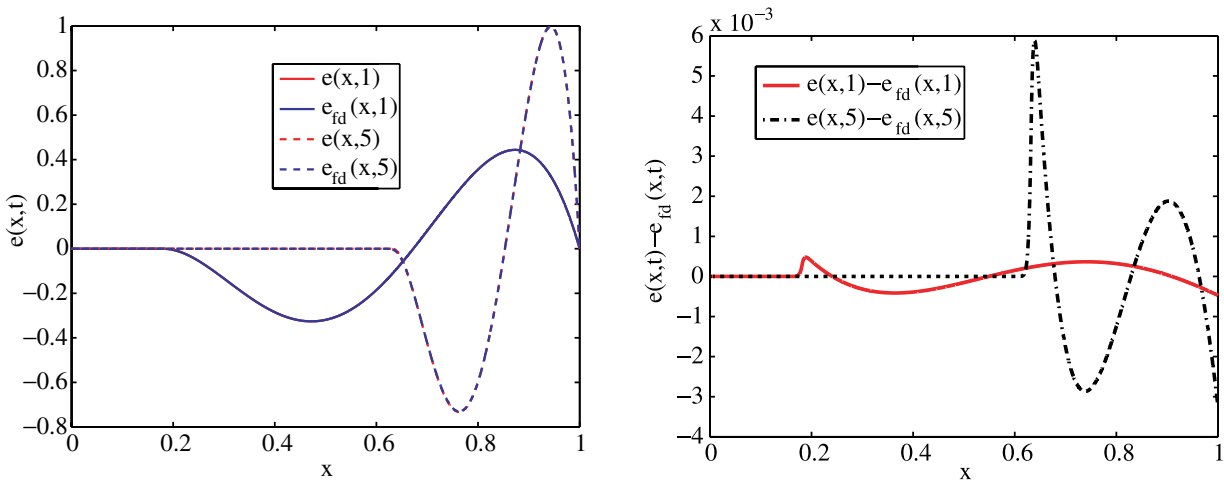

FIG. 5. (left): Analytical solution and approximate solution for sensitivity with respect to $g$ at $t=1$ and $t=5$. (right): Error between analytical solution and approximate solution at $t=1$ and $t=5$. 


\section{REFERENCES}

[1] H. M. Adelman and R.T. Haftka, Sensitivity analysis of discrete structural systems, A.I.A.A. Journal, 24 (1986), 823-832.

[2] R. Bellman and K. L. Cooke, Differential-Difference Equations, Academic Press, New York, 1963. MR0147745 (26:5259)

[3] H. T. Banks and F. Kappel, Transformation semigroups and $L^{1}$-approximation for size-structured population models, Semigroup Forum, 38 (1989), 141-155. MR976199 (90b:92040)

[4] H. T. Banks, F. Kappel and C. Wang, Weak solutions and differentiability for size-structured population models, International Series of Numerical Mathematics, 100 (1991), 35-50. MR1155635 (93f:92020)

[5] H. T. Banks, F. Kojima and W. P. Winfree, Boundary estimation problems arising in thermal tomography, Inverse Problems, 6 (1990), 897-921. MR1082231 (91k:80003)

[6] H. T. Banks and H. K. Nguyen, Sensitivity of dynamical systems to Banach space parameters, Journal of Mathematical Analysis and Applications, 323 (2006), 146-161. MR2261157 (2007g:34118)

[7] H. T. Banks, V. A. Bokil, S. Hu, A. K. Dhar, R. A. Bullis, C. L. Browdy and F.C.T. Allnutt, Modeling shrimp biomass and viral infection for production of biological countermeasures, Mathematical Biosciences and Engineering, 3 (2006), 635-660. MR2249893

[8] H. T. Banks, M. Davidian and J.R. Samuels, Jr., An inverse problem statistical methodology summary, CRSC-TR07-14, August, 2007.

[9] H. T. Banks, S. Dediu and H. K. Nguyen, Sensitivity of dynamical systems to parameters in a convex subset of a topological vector space, Mathematical Biosciences and Engineering, 4 (2007), 403-430. MR2328091

[10] H. T. Banks, S. L. Ernstberger and S. L.Grove, Standard errors and confidence intervals in inverse problems: Sensitivity and associated pitfalls, J. Inverse and Ill-Posed Problems, 15 (2007), 1-18. MR2313504 (2007m:34195)

[11] J. J. Batzel, F. Kappel, D. Schneditz and H. T. Tran, Cardiovascular and Respiratory Systems: Modeling, Analysis and Control, SIAM Frontiers in Applied Math., SIAM, Philadelphia, 2006. MR2279586(2007g:92039)

[12] K. P. Burnham and D. R. Anderson, Model Selection and Multimodal Inference: A Practical Information-Theoretic Approach, Springer-Verlag, New York, NY, 2002. MR.1919620

[13] J. A. Burns, T. Lin and L. Stanley, A Petrov-Galerkin finite element method for interface problems arising in sensitivity computations, Computers and Mathematics with Applications, 49 (2005), 1889-1903. MR2154692(2006c:65069)

[14] J. A. Burns, D. Rubio and M. I. Troparevsky, Sensitivity computations for elliptic equations with interfaces, in Proceedings ICNPAA-2006 Conference on Mathematical Problems in Engineering and Aerospace Sciences, Cambridge Sci. Publ., Cambridge, 2007. MR2402293

[15] J. A. Burns and L. Stanley, A note on the use of transformations in sensitivity computations for elliptic systems, Mathematical and Computer Modelling, 33 (2001), 101-114. MR.1812544

[16] J. Borggaard and J. A. Burns, A PDE sensitivity equation method for optimal aerodynamic design, Journal of Computational Physics, 136 (1997), 366-384. MR1474410 (98i:76047)

[17] J. Borggaard and A. Verma, On efficient solutions to the continuous sensitivity equation using automatic differentiation, SIAM Journal on Scientific Computing, 22 (2001), 39-62. MR.1769486 (2001e:49071)

[18] A. Calsina and J. Saldaña, A model of physiologically structured population dynamics with a nonlinear individual growth rate, Journal of Mathematical Biology, 33 (1995), 335-364. MR1320428 (96i:92020)

[19] G. Casella and R. L. Berger, Statistical Inference, Duxbury, California, 2002.

[20] J. B. Cruz, ed., System Sensitivity Analysis, Dowden, Hutchinson \& Ross, Inc., Stroudsburg, PA, 1973. MR0392045 (52:12863)

[21] M. Davidian and D. M. Gilitan, Nonlinear Models for Repeated Measurement Data, Chapman \& Hall, London, 1995.

[22] M. C. Delfour and J. P. Zolesio, Anatomy of the shape Hessian, Annali di Matematica pura ed applicata, 159 (1991), 315-339. MR1145103(92k:35016)

[23] M.C. Delfour and J. P. Zolesio, Shapes and Geometries. Analysis, Differential Calculus, and Optimization, SIAM, Philadelphia, 2001. MR1855817(2002i:49002) 
[24] M. Eslami, Theory of Sensitivity in Dynamic Systems: An Introduction, Springer-Verlag, Berlin, 1994. MR 1318439 (96c:93001)

[25] P. M. Frank, Introduction to System Sensitivity Theory, Academic Press, Inc., New York, NY, 1978. MR0496987 (58:15413)

[26] J. Haslinger and R. A. E. Mäkinen, Introduction to Shape Optimization: Theory, Approximation and Computation, SIAM, Philadelphia, 2003. MR.1969772 (2004d:49001)

[27] E. J. Haug, K. K. Choi and V. Komkov, Design Sensitivity Analysis of Structural Systems, Academic Press, New York, NY, 1986. MR860040 (89i:73061)

[28] M. Kleiber, H. Antunez, T. D. Hien and P. Kowalczyk, Parameter Sensitivity in Nonlinear Mechanics: Theory and Finite Element Computations, John Wiley \& Sons, New York, NY, 1997.

[29] K. Ito, F. Kappel and G. Peichl, A fully discretized approximation scheme for size-structured population models, SIAM Journal on Numerical Analysis, 28 (1991), 923-954. MR1111447 (92f:92029)

[30] D. Jiang, A. L. Lawrence, W. H. Neil and H. Gong, Effects of temperature and salinity on nitrogenous excretion by Litopenaeus vannamei juveniles, Journal of Experimental Marine Biology and Ecology, 253 (2000), 193-209.

[31] M. Kot, Elements of Mathematical Ecology, Cambridge University Press, Cambridge, 2001. MR 2006645 (2004e:92033)

[32] J. A. J. Metz and O. Diekmann (eds.), The Dynamics of Physiologically Structured Populations, Lecture Notes in Biomathematics, 68, Springer, 1986. MR860959 (88b:92049)

[33] A. G. McKendrick, Applications of mathematics to medical problems, Proceedings of the Edinburgh Mathematical Society, 40 (1926), 98-130.

[34] O. Pironneau, Optimal Design for Elliptic Systems, Springer-Verlag, New York, 1984. MR725856 (86e:49003)

[35] A. Saltelli, K. Chan and E. M. Scott, eds., Sensitivity Analysis, Wiley Series in Probability and Statistics, John Wiley \& Sons, New York, NY, 2000. MR.1886391 (2003c:62008)

[36] G. A. F. Seber and C. J. Wild, Nonlinear Regression, John Wiley \& Sons, Inc., New York, 1989. MR 986070 (90j:62004)

[37] J. W. Sinko and W. Streifer, A new model for age-size structure of a population, Ecology, 48 (1967), 910-918.

[38] L. G. Stanley and D. L. Stewart, Design Sensitivity Analysis: Computational Issues of Sensitivity Equation Methods, Frontiers in Applied Mathematics, vol. 25, SIAM, Philadelphia, 2002. MR:1946496 (2003j:49003)

[39] H. von Foerster, Some remarks on changing populations, in The Kinetics of Cellular Proliferation, F. Stohlman, Jr., ed., Grune \& Stratton, New York, 1959, pp. 382-407.

[40] K. Thomaseth and C. Cobelli, Generalized sensitivity functions in physiological system identification, Annals of Biomedical Engineering, 27 (1999), 607-616.

[41] A. Wierzbicki, Models and Sensitivity of Control Systems, Studies in Automation and Control 5, Elsevier Science Publ. Co., Inc., New York, NY, 1984.

[42] P. V. Wyk and J. Scarpa, Water quality requirement and management, http://www.hboie.du/aqua/ downloads/pdf/shrimpmanual_chapter8.pdf. 